\title{
Information Spillovers in Asset Markets with Correlated Values
}

\author{
By Vladimir Asriyan, William Fuchs, and Brett Green*
}

\begin{abstract}
We study information spillovers in a dynamic setting with correlated assets owned by privately informed sellers. In the model, a trade of one asset can provide information about the value of other assets. Importantly, the information content of trading behavior is endogenously determined. We show that this endogeneity leads to multiple equilibria when assets are sufficiently correlated. The equilibria are ranked in terms of both trade volume and efficiency. The model has implications for policies targeting post-trade transparency. We show that introducing post-trade transparency can increase or decrease welfare and trading volume depending on the asset correlation, equilibrium being played, and the composition of market participants. (JEL D82, D83, G14, G18)
\end{abstract}

When asset values are correlated and sellers are privately informed, a trade of one asset can be informative about the value of other assets, which can in turn influence the trading decision of others. These "information spillovers" can play an important role in determining the manner and the efficiency with which assets are reallocated.

In this paper, we develop a stylized framework to understand the role of information spillovers. The model involves two sellers (for convenience, we refer to them as Ann and Bob), each with an indivisible asset that has a value which is either low or high. Each seller is privately informed about the value of their asset. There is common knowledge of gains from trade, but buyers face a lemons problem (Akerlof

\footnotetext{
*Asriyan: CREI, Ramon Trias Fargas, 25-27, Merce Rodoreda Bldg., Barcelona, 08005, Spain (email: vasriyan@crei.cat); Fuchs: Austin McCombs School of Business, University of Texas, 2110 Speedway, Austin, TX 78705, and Universidad Carlos III Madrid, Calle Madrid, 126, 28903 Getafe, Spain (email: wfuchs@gmail.com); Green: Haas School of Business, University of California, 2220 Piedmont Avenue, Berkeley, CA 94720 (email: greenb@berkeley.edu).Asriyan acknowledges support from the Spanish Ministry of Economy and Competitiveness Grant (ECO2014-54430-P) and the Barcelona GSE Seed Grant. Fuchs gratefully acknowledges support from the ERC Grant 681575. We thank Fernando Broner, Brendan Daley, Darell Duffie, Satoshi Fukuda, Harrison Hong, Boyan Jovanovic, Ayca Kaya, Vincent Maurin, Christine Parlour, Larry Samuelson, Andy Skrzypacz, Victoria Vanasco, Jaume Ventura, and seminar participants at Banque de France, Bocconi University, CREi, INSEAD, Kellogg School of Management, London School of Economics, New York University, Olin Business School at Washington University, UNC Chapel Hill, University of Minnesota, USC, Yale University, San Francisco Federal Reserve, Sciences Po, University of Colorado Leeds School of Business, Stanford Graduate School of Business, Swiss Finance Institute, University of Minnesota, UT Austin, University of Washington Foster School of Business, as well as conference participants at the Finance Theory Group, 14th Annual Columbia/Duke/MIT/Northwestern IO Theory Conference, SAET meetings in Rio, SED meetings in Warsaw, Workshop on Information Frictions in Macro at TSE, Workshop on Economic Policy and Financial Frictions at EUI, and the 11th World Congress of the Econometric Society in Montreal. The authors declare that they have no relevant or material financial interests that relate to the research described in this paper.

Go to https://doi.org/10.1257/aer.20151714 to visit the article page for additional materials and author disclosure statement(s).
} 
1970). Trading takes place via a competitive market over the course of two periods. In the first period, potential buyers can approach a seller and make offers. If a seller rejects all offers in the first period, then she can entertain more offers from new buyers in the second period. In this setting, inefficiencies can arise from delays in trade or a failure to trade altogether.

The key ingredient of our model is that the asset values are positively correlated. Provided that transactions are observable, a trade of one asset (or lack thereof) can provide information to buyers about the value of the other asset. Importantly, the information content of observed trading behavior is endogenous. To understand the nature of the endogeneity, suppose that in the first period, Bob trades with a high probability if he owns a low-value asset and does not trade if he owns a high-value asset. Then, because the asset values are correlated, observing whether Bob trades has information content about the value of Ann's asset. On the other hand, if Bob plans to sell the asset in the first period regardless of its value, then observing him trade is completely uninformative about the quality of Ann's asset.

Because Bob's trading strategy influences the amount of information revealed about Ann's asset, it effects her decision of when to sell, which in turn influences the amount of information revealed about Bob's asset and thus also effects his decision. This strategic interaction is the primary mechanism underlying our results.

In equilibrium, low-value assets are more likely to trade in the first period. ${ }^{1}$ Therefore, observing a transaction in the first period is "bad news" about the other asset. The strategic interaction can be decomposed into two separate effects, which we refer to as the bad news and good news effects. The bad news effect is that, as Bob trades more aggressively, it becomes more likely from Ann's perspective that bad news will be revealed, which therefore induces Ann to trade more aggressively. The good news effect is that, conditional on not observing a trade by Bob, the market's belief about Ann's asset is more favorable, which leads to higher prices and induces Ann to trade less aggressively.

When the bad news effect dominates, there is a strategic complementarity between the sellers. The more likely Bob is to accept a low offer, the more inclined Ann becomes to also accept a low offer. When the good news effect dominates, just the opposite relationship obtains. It turns out that the good news effect dominates when Bob uses a less aggressive strategy while the bad news effect dominates when Bob uses a more aggressive strategy. Hence, the optimal trading behavior of Ann is non-monotonic (first decreasing and eventually increasing) in Bob's behavior.

After characterizing the nature of the strategic interaction, we show that all equilibria must be symmetric and feature strictly positive probability of trade in both periods (Proposition 3). We then establish our main result (Theorem 1), which states that when information spillovers are strong (correlation is high), there are three equilibria. The equilibria are ranked both in terms of the volume of trade that takes place and the total welfare. The higher is the volume of trade in the first period, the more efficiently assets are reallocated and the higher is total welfare. Interestingly, comparative statics with respect to the strength of information spillovers vary across

\footnotetext{
${ }^{1}$ This feature is common in dynamic models with adverse selection and often referred to as the "skimming" property.
} 
equilibria. Thus, increasing the level of correlation between assets can lead to higher or lower trading volume and welfare depending on which equilibrium is played.

To provide intuition as to why this multiplicity obtains, consider the extreme case in which the assets are perfectly correlated. Suppose that Bob trades with probability one if he owns a low-type asset and with probability zero otherwise. If Ann delays trade in the first period, then her type will be perfectly revealed by whether Bob trades. Conditional on observing a trade by Bob in the first period, buyers will correctly infer that Ann has a low-value asset and offer a low price in the second period. Therefore, Ann has no incentive to delay trade if she has a low-value asset and strictly prefers to trade in the first period. Hence, there exists an equilibrium in which both low-value assets trade with probability one in the first period.

Next, suppose that Bob trades with some intermediate probability in the first period if he owns a low-type asset and with probability zero otherwise. From Ann's perspective, there is still positive probability that her type will be revealed if buyers observe a trade by Bob (the bad news effect), but there is also some chance that Bob does not trade, in which case buyers correctly infer that Ann is more likely to have a good asset making them willing to offer a high price in the second period (the good news effect). The potential for getting a high price in the second period makes Ann indifferent between trading in the first period, and hence she is willing to trade with some intermediate probability. Thus, there also exists an equilibrium in which both low-value assets trade with an intermediate probability in the first period.

Though our model is stylized and abstracts from institutional details of specific markets, the economic forces we identify could apply to a variety of asset markets, both real and financial. For example, the assets could refer to different tranches of an asset-backed security or corporate debt issuance, where both the issuer and certain investors have access to private information about the underlying collateral or firm, which was not reliably disclosed. Allegations along these lines were made against numerous banks and asset managers in the aftermath of the financial crisis. ${ }^{2}$ Another possible application is real estate markets, where sellers of nearby homes have private information about the desirability of the location and neighborhood trends. Indeed, Kurlat and Stroebel (2015) provide evidence that private information about neighborhood characteristics affects home prices, while Adelino, Gerardi, and Hartman-Glaser (2016) document evidence consistent with a dynamic adverse selection problem in secondary markets for mortgages.

The extent to which information spillovers can impact market behavior depends crucially on the ability of agents to observe previous transactions. In recent years, websites such as Zillow, RedFin, and Trulia have drastically increased the degree of transparency in real estate markets (Richardson and Zumpano 2012). Potential buyers and sellers now have instantaneous access to the entire history of recent transactions, whereas previously this information was only easily accessible to brokers..$^{3}$ Alti, Engelberg, and Parsons (2011) present direct evidence of information spillovers in real estate markets that is consistent with our theoretical predictions. They find that a sale of one home increases the probability that a neighboring home

\footnotetext{
${ }^{2}$ See www.sec.gov/spotlight/enf-actions-fc.shtml for a list of such allegations.

${ }^{3}$ See Gordon (2012) for a broader overview of how transparency in real estate markets has increased worldwide in recent years.
} 
sells in the near future even after controlling for demand. These effects are strongest in homogeneous markets (where presumably the correlation between houses is higher) and can reduce time-on-the-market by as much as 20 percent.

Post-trade (or transactional) transparency has also received significant attention from policymakers in financial markets. In July 2002, the corporate bond market underwent a significant change when regulators implemented Transactions Reporting and Compliance Engine (TRACE), which mandated that prices and volume of completed transactions be publicly disclosed. Since then, TRACE has been expanded to include other asset classes. There are also ongoing efforts by regulators to increase transparency in the markets for numerous derivatives (Title VII of Dodd-Frank) and European corporate bonds (Learner 2011).

To explore the implications of post-trade transparency, we modify the model slightly so that buyers only observe transactions on the platform in which they participate. Introducing transactional transparency in such an environment can lead to equilibrium multiplicity. Depending on which equilibrium is played, trading volume can increase or decrease and welfare can increase or remain unchanged. Our findings thus can help explain why Bessembinder, Maxwell, and Venkataraman (2006) and Edwards, Harris, and Piwowar (2007) find that market participants gain from the introduction of TRACE, while Goldstein, Hotchkiss, and Sirri (2007) see no effect within a subclass of securities, and Asquith, Covert, and Pathak (2013) find significantly different results for bonds that were part of the different phases of the TRACE program.

Regulators often argue that transactional transparency "creates a level playing field for all investors" (NASD 2005). To investigate this claim, we extend our model to a setting where an insider (e.g., a broker-dealer) has better access to transaction data than others (e.g., retail or institutional investors). We confirm that post-trade transparency indeed reduces the trading profits of the insider, which may help explain broker-dealers' resistance to the proposed changes. Mandating post-trade transparency also mitigates the trading losses of investors who are naïve about the fact that they face competition from an insider. However, if investors are sophisticated, then post-trade transparency has no effect on their welfare and can lead to an overall reduction in efficiency relative to an opaque market. Thus, "leveling the playing field" may come at a cost and the desirability of such a policy depends on the composition of market participants.

Related Literature.- Our work is related to Daley and Green (2012, 2016), who study a setting in which information is exogenously revealed to uninformed buyers. They show that when information (or news) quality is exogenous, the unique equilibrium involves periods of no trade. In contrast, we show that when information is endogenously revealed by the trading behavior of other market participants, there can exist multiple equilibria all of which require trade to occur with strictly positive probability in each period.

The transparency of offers has been studied by Nöldeke and van Damme (1990), Swinkels (1999), Hörner and Vieille (2009), and Fuchs, Öry, and Skrzypacz (2016). Our work differs in two important respects. First, these papers consider a setting with a single seller, whereas we explore the strategic considerations of multiple sellers with correlated assets. Second, they consider pre-trade transparency with respect 
to offers that are made, whereas we consider post-trade transparency with respect to transactions that have taken place. The two types of transparency have different implications. We find transaction transparency can lead to multiple equilibria and weakly improves efficiency (with symmetric buyers), whereas offer transparency typically reduces efficiency.

There is also a large literature within accounting and finance studying the effect of public disclosure of firm specific information. Healy and Palepu (2001) and Verrecchia (2001) provide surveys of both the theoretical and empirical work in this area. One key difference is that this literature takes the information to be disclosed as given and studies the effects of whether, when, how much, and how frequently it is made public. Naik, Neuberger, and Viswanathan (1999) study trade disclosure in a (Kyle 1985) setting with risk averse agents. They show that disclosure in the first trading period reduces private information in the second trading period, but can increase price volatility and thus has ambiguous welfare effects. In our setting, agents are risk neutral yet public disclosure of trades can still lead to worse outcomes when buyers are asymmetrically informed because it increases competition (and thus prices) giving the seller more incentive to (inefficiently) delay trade.

Kaya and Kim (2015) analyze a model with a single seller in which sequential buyers receive a private signal about the value of the asset. Instead, our focus is on the two-way interaction between trade and the information generated by it. The idea of a two-way feedback between trading activity and market informativeness is also present in Cespa and Vives (2015). They study a noisy rational expectations model and find that multiple equilibria can arise when noise-trader shocks are sufficiently persistent and informed buyers care only about their short-term returns. While our approaches are substantially different, their model also delivers equilibria that have high trading volume and market informativeness as well as equilibria in which trading volume and informativeness are low.

Finally, Drugov $(2010,2014)$ considers the related problem of information externalities among two bargaining pairs. There are two important differences with respect to this work. First, he considers a different market structure where there is only one buyer per seller rather than competing buyers. Second, in his model the value of the seller is independent of the value of the buyer, while in ours the values are correlated giving rise to a lemons problem.

The rest of the paper is organized as follows. In Section I, we present the basic ingredients and conduct preliminary analysis. In Section II, we characterize the equilibria of the model and explore the effect of asset correlation on trading volume and welfare. In Section III, we explore the implications of post-trade transparency in opaque markets. Section IV concludes. All proofs are in the Appendix.

\section{The Model}

There are two sellers, indexed by $i \in\{A, B\}$. Each seller owns one indivisible asset and is privately informed of her asset's type, denoted by $\theta_{i} \in\{L, H\} .4$ Seller $i$ has a value $c_{\theta}$ for a type- $\theta$ asset, where $c_{L}<c_{H}$. The market for assets is

\footnotetext{
${ }^{4}$ The model and the results can be extended to an environment with an arbitrary number of sellers, as we show in Asriyan, Fuchs, and Green (2016).
} 
competitive-there are at least two identical buyers competing for assets in each period ${ }^{5}$ The value of a type- $\theta$ asset to a buyer is $v_{\theta}$ and there is common knowledge of gains from trade, $v_{\theta}>c_{\theta}$, which can be motivated by, for example, preference shocks, liquidity constraints, or hedging demands.

There are two trading periods: $t \in\{1,2\}$. In each period, buyers can make offers to each seller. A buyer whose offer is rejected gets a payoff of zero and exits the game. ${ }^{6}$ The payoff to a buyer who purchases an asset of type $\theta$ at price $p$ is given by

$$
v_{\theta}-p
$$

Sellers discount future payoffs by a discount factor $\delta \in(0,1)$. The payoff to a seller with an asset of type $\theta$, who agrees to trade at a price $p$ in period $t$ is

$$
\left(1-\delta^{t-1}\right) c_{\theta}+\delta^{t-1} p
$$

If the seller does not trade at either date, his payoff is $c_{\theta}$. All players are risk neutral. One can interpret $c_{\theta}$ and $v_{\theta}$ as the present value of the flow payoffs from owning the asset to the seller and buyer, respectively. $]^{7}$ That is, $c_{\theta}=\sum_{t=1}^{\infty} \delta^{t-1} x_{\theta}$ $=(1-\delta)^{-1} x_{\theta}$, where $x_{\theta}$ is the seller's expected flow payoff from owning a type- $\theta$ asset for one period.

The key feature of our model is that asset values are positively (but imperfectly) correlated. To model this correlation, let the unconditional distribution of $\theta_{i}$ be given by

$$
\operatorname{Pr}\left(\theta_{i}=L\right)=1-\pi
$$

where higher $\pi \in(0,1)$ corresponds to more favorable beliefs. The ex ante distribution of $\theta_{i}$ conditional on $\theta_{j}$ is

$$
\operatorname{Pr}\left(\theta_{i}=L \mid \theta_{j}=L\right)=\lambda
$$

for some $\lambda \in(1-\pi, 1) \cdot 8$

\footnotetext{
${ }^{5}$ Perfect competition among buyers is not essential for our results. In Section IIIB, we allow for asymmetries among buyers in which case the buyer side of the market makes positive profits.

${ }^{6}$ The assumption that buyers are "short-lived" (i.e., make offers in only one period) is, by now, fairly standard in this literature (e.g., Swinkels 1999; Kremer and Skrzypacz 2007; Hörner and Vieille 2009). The equilibria we construct are also equilibria in a model with long-lived buyers if offers are publicly observable. However, if offers are not observable, then a long-lived buyer has incentive to engage in a form of experimentation by submitting an offer that loses money in the first period (if it is accepted) in order to make a profitable one in the second period. Note that this issue arises even without multiple sellers and correlated assets.

${ }^{7}$ The fact that one asset sold or the timing of its sale does not change the intrinsic valuation for potential buyers of the other asset. Although these might be important considerations in some applications, we eliminate them from our analysis since our objective is to isolate the effects stemming from information spillovers.

${ }^{8}$ With perfect correlation (i.e., $\lambda=1$ ), the set of equilibria is sensitive to the specification of off-equilibrium path beliefs. Nevertheless, in the online Appendix we show that the set of equilibria with perfect correlation is the limit of the set of equilibria as $\lambda \rightarrow 1$.
} 
Importantly, asset correlation introduces the possibility that a trade of one asset contains relevant information about the value of the other asset. 9 To capture this possibility, we assume that any transaction that occurs in the first period is observed by buyers prior to when they make their offers in the second period. For convenience, we also assume that offers are made privately; the level of rejected offers is not observed by other buyers. 10

Our primary interest is to explore how the correlation of asset values influences equilibrium trading behavior. To do so, we focus on primitives which satisfy the following assumptions:

ASSUMPTION 1: $\pi v_{H}+(1-\pi) v_{L}<c_{H}$.

ASSUMPTION 2: $v_{L}<(1-\delta) c_{L}+\delta c_{H}$.

The first assumption, which we refer to as the "lemons" condition, asserts that the adverse selection problem is severe enough to rule out the first-best equilibrium in which both sellers trade in the first period with probability one (w.p.1) regardless of their type. If the lemons condition does not hold, then the first-best outcome is an equilibrium. There can also exist another equilibrium in which trade is not fully efficient. Thus, information spillovers can distort an otherwise efficient market by introducing the possibility of learning and provide incentives for the high type to wait for a better price. 11

The second assumption rules out the fully separating equilibrium in which the low type trades in the first period w.p. 1 and the high type trades in the second period w.p.1. Together, these two conditions rule out trivial equilibria in which information spillovers are irrelevant for equilibrium behavior. ${ }^{12}$

\section{A. Strategies, Information Sets, and "News"}

A strategy of a buyer is a mapping from his information set to a probability distribution over offers. In the first period (i.e., at $t=1$ ), a buyer's information set is empty. In the second period, buyers know whether each asset traded in the first period. If asset $i$ trades in the first period, then it is efficiently allocated and it is without loss to assume that buyers do not make offers for it in the second period (Milgrom and Stokey 1982). If asset $i$ does not trade in the first period, buyers update their beliefs about $\theta_{i}$ based on two pieces of information: (i) the fact that seller $i$ did not trade in the first period and (ii) whether seller $j$ traded in the first period.

The second piece of information or "news" is commonly observed across all buyers and is denoted by $z_{i} \in\{b, g\}$ (the reason for this notation will soon become

\footnotetext{
${ }^{9}$ For parsimony, we assume the type remains fixed over time. Our results can be generalized to a setting where asset types evolve over time (e.g., due to aggregate shocks). In such a setting, the relevance of information spillovers hinges on the lagged cross correlation between asset values, i.e., $\operatorname{corr}\left(\theta_{j, t+1}, \theta_{i, t}\right)$.

${ }^{10}$ Fuchs, Öry, and Skrzypacz (2016) show that this specification is without loss in a setting with a single asset.

${ }^{11}$ This finding is similar to results in Daley and Green (2012) in which the introduction of exogenous news can reduce overall efficiency.

${ }^{12}$ To rule out fully separating equilibria only requires $v_{L}<(1-\delta) c_{L}+\delta v_{H}$; our stronger Assumption 2 further simplifies exposition without affecting our main results.
} 
apparent). If $z_{i}=b$, then seller $j$ traded in the first period. If $z_{i}=g$, then seller $j$ rejected all offers in the first period.

Remark 1: Our results remain unchanged if buyers also observe transaction prices. Because buyers are uninformed and make the offers, no additional information (beyond whether a transaction occurred) is revealed by the price.

The strategy of each seller is a mapping from her information set to a probability of acceptance. Seller $i$ 's information includes her type, her previous and current offers, as well as the information set of buyers.

\section{B. Equilibrium Concept}

We use Perfect Bayesian Equilibria (PBE) as our equilibrium concept. This has three implications. First, each seller's acceptance rule must maximize her expected payoff at every information set taking buyers' strategies and the other seller's acceptance rule as given (seller optimality). Second, any offer in the support of a buyer's strategy must maximize his expected payoff given his beliefs, other buyers' strategies and the seller's strategy (buyer optimality). Third, given their information set, buyers' beliefs are updated according to Bayes' rule whenever possible (belief consistency).

\section{Preliminary Analysis}

It is convenient to establish some basic properties all equilibria must satisfy. Because there are multiple buyers, their individual offers are not uniquely pinned down by PBE. We refer to the bid for asset $i$ at time $t$ as the maximal offer made across all buyers for asset $i$ at time $t$.

Let $V(\tilde{\pi}) \equiv \tilde{\pi} v_{H}+(1-\tilde{\pi}) v_{L}$ denote buyer's expected value for an asset given an arbitrary belief $\tilde{\pi}$. Let $\bar{\pi} \in(\pi, 1)$ be such that $V(\bar{\pi})=c_{H}$, and let $\pi_{i}$ denote the probability that buyers assign to $\theta_{i}=H$ just prior to making offers in the second period. In equilibrium, $\pi_{i}$ is determined by belief consistency and the realization of news. Taking these beliefs as given, equilibrium play at $t=2$ corresponds to that of the familiar static market for lemons.

LEMMA 1: If seller $i$ does not trade in the first period then, in the second period:

(i) if $\pi_{i}<\bar{\pi}$, then the bid is $v_{L}$ and seller $i$ accepts only if $\theta_{i}=L$;

(ii) if $\pi_{i}>\bar{\pi}$, then the bid is $V\left(\pi_{i}\right)$ and seller $i$ accepts with probability one;

(iii) if $\pi_{i}=\bar{\pi}$, then the bid is $c_{H}=V\left(\pi_{i}\right)$ with some probability $\phi_{i} \in[0,1]$ and $v_{L}$ otherwise.

A high-type seller will only accept a bid greater than $c_{H}$. Therefore, when the expected value of the asset is below $c_{H}$ (i.e., $\pi_{i}<\bar{\pi}$ ), there is no way for a buyer to attract a high-type seller without making a loss. Thus, buyers will trade only with the low types and competition pushes the bid price to $v_{L}$, implying (i). When the 
expected value is above $c_{H}$ (i.e., $\pi_{i}>\bar{\pi}$ ), competition between buyers forces the equilibrium offer to be the expected value, implying (ii). In (iii), the expected value of the asset is exactly $c_{H}$ and hence buyers are indifferent between offering $c_{H}$ and trading with both types or offering $v_{L}$ and only trading with the low type.

Continuation Values.-It follows from Lemma 1 that for a given belief and buyer mixing probability for asset $i,\left(\pi_{i}, \phi_{i}\right)$, the payoff to a high-type seller $i$ in the second period is

$$
F_{H}\left(\pi_{i}, \phi_{i}\right) \equiv \max \left\{c_{H}, V\left(\pi_{i}\right)\right\}
$$

and a low type's payoff in the second period is given by

$$
F_{L}\left(\pi_{i}, \phi_{i}\right) \equiv \begin{cases}v_{L} & \text { if } \pi_{i}<\bar{\pi} \\ \phi_{i} c_{H}+\left(1-\phi_{i}\right) v_{L} & \text { if } \pi_{i}=\bar{\pi} \\ V\left(\pi_{i}\right) & \text { if } \pi_{i}>\bar{\pi}\end{cases}
$$

Figure 1 illustrates how these payoffs depend on $\pi_{i}$ and $\phi_{i}$. Notice that if $\pi_{i}=\bar{\pi}$, the low-type seller's payoff is not uniquely pinned down as it depends on the likelihood that $c_{H}$ is offered. Hence, $F_{L}$ can take values in the interval $\left[v_{L}, c_{H}\right]$ if $\pi_{i}=\bar{\pi}$. On the other hand, $F_{H}$ is independent of the probability that $c_{H}$ is offered.

Given her information set in the first period, the seller $i$ 's payoff in the second period is stochastic because buyers' beliefs will depend on the news about asset $j$ and because buyers may mix over offers. Fixing a candidate equilibrium, the expected continuation value from rejecting the bid in the first period for seller $i$ of type $\theta$ is

$$
Q_{\theta}^{i}=(1-\delta) \cdot c_{\theta}+\delta \cdot E_{\theta}\left\{F_{\theta}\left(\pi_{i}, \phi_{i}\right)\right\}
$$

LEMMA 2: In any PBE, the expected continuation value for the high type is strictly greater than that for the low type: $Q_{H}^{i}>Q_{L}^{i}$.

This is due to (i) $F_{H} \geq F_{L}$; (ii) the flow payoff to a high type from delay is higher; and (iii) a high type rationally believes it is less likely that bad news will arrive and thus she expects a (weakly) better distribution of price offers in the second period. Note that (iii) is true regardless of the first-period trading strategies used by the other seller. That is, any "good" news is more likely if $\theta_{i}=H$ than if $\theta_{i}=L$ and conversely.

Consider now the seller's decision in the first period. The strict ranking of continuation values implies that if a high type is willing to accept the bid in the first period, then a low type will strictly prefer to accept. By Assumption 1, buyers' prior beliefs are sufficiently pessimistic to rule out an offer weakly above $c_{H}$. Thus, buyers make offers that only a low-type seller will accept.

LEMMA 3: In the first period, the equilibrium outcome satisfies the following:

(i) The bid for asset $i$ is $v_{L}$. 


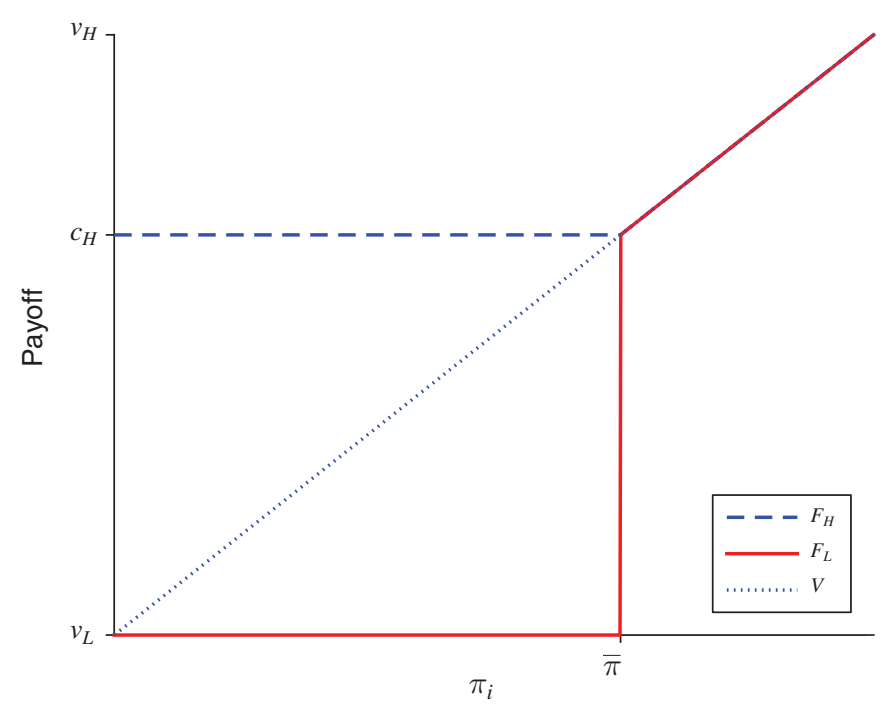

Figure 1. The Set of Second-Period Payoffs of the Seller as They Depend on $\theta_{i}$ And $\pi_{i}$

(ii) A high-type seller rejects the bid. A low-type seller accepts with probability $\sigma_{i} \in[0,1)$.

To see why $\sigma_{i}$ must be strictly less than one, suppose to the contrary that $\sigma_{i}=1$. Then conditional on rejecting the offer, belief consistency requires that buyers believe seller $i$ is a high type w.p.1 (regardless of $z_{i}$ ) and thus the bid must be $v_{H}$ in the second period (by Lemma 1). But, then a low-type seller would get a higher payoff by not trading in the first period (see Assumption 2), violating optimality of the seller's strategy. Thus, it must be that $\sigma_{i} \in[0,1)$.

Updating.-As highlighted above, buyers' beliefs in the second period determine equilibrium play. There are two ways in which the prior is updated between the first and second periods. First, conditional on seller $i$ rejecting the offer in the first period, buyers' interim belief is given by

$$
\pi_{\sigma_{i}} \equiv \operatorname{Pr}\left(\theta_{i}=H \mid \text { reject at } t=1\right)=\frac{\pi}{\pi+(1-\pi)\left(1-\sigma_{i}\right)}
$$

Second, before making their offers in the second period, buyers learn about any trades that took place in the first period. Since asset values are positively correlated and only low types trade in the first period, news that asset $j$ sold (i.e., $z_{i}=b$ ) will lead to a negative revision in beliefs, and $z_{i}=g$ will lead to a positive revision.

Exactly how this news is incorporated into the posterior depends on the trading strategy of seller $j$. It is useful to define first the probability of news $z_{i}$ conditional on the type $\theta_{i}$ of seller, which we denote by $\rho_{\theta}^{i}\left(z_{i}\right)$. Specifically, the probability of observing the event $z_{i}=b$, given seller $i$ is of type $\theta$ is

$$
\rho_{\theta}^{i}(b) \equiv \operatorname{Pr}\left(z_{i}=b \mid \theta_{i}=\theta\right)=\sigma_{j} \cdot \operatorname{Pr}\left(\theta_{j}=L \mid \theta_{i}=\theta\right) .
$$


Provided $\sigma_{j}>0$, we can use equations (4) and (5) to express the posterior probability of seller $i$ being high type after news $z$ as

$$
\begin{aligned}
\pi_{i}\left(z ; \sigma_{i}, \sigma_{j}\right) & \equiv \operatorname{Pr}\left(\theta_{i}=H \mid i \text { rejects at } t=1, z_{i}=z\right) \\
& =\frac{\pi_{\sigma_{i}} \cdot \rho_{H}^{i}(z)}{\pi_{\sigma_{i}} \cdot \rho_{H}^{i}(z)+\left(1-\pi_{\sigma_{i}}\right) \cdot \rho_{L}^{i}(z)}
\end{aligned}
$$

To conserve on notation, we often suppress arguments of $\pi_{i}$. Notice that $\pi_{i}(z)$ has the expected property that $\pi_{i}(b) \leq \pi_{\sigma_{i}} \leq \pi_{i}(g) \cdot{ }^{13}$ A few additional properties are worth noting. First, $\pi_{i}(b)$ is increasing in $\sigma_{i}$ and is independent of $\sigma_{j}$. The latter follows because only a low-type seller $j$ trades in the first period and therefore upon observing $z_{i}=b$, buyers know that $\theta_{j}=L$ regardless of how aggressively seller $j$ trades. On the other hand, $\pi_{i}(g)$ is increasing in both $\sigma_{i}$ and $\sigma_{j}$, since a more aggressive trading strategy for seller $j$ implies a lower likelihood of $z_{i}=g$. Finally, $\pi_{i}(g)$ is more sensitive to changes in $\sigma_{i}$ than $\sigma_{j}$ since seller $i$ 's own trading strategy is always (weakly) more informative about her type than is seller $j$ 's.

\section{Equilibrium}

From Lemmas 1 and 3 as well as the updating summarized by equations (4)-(6), an equilibrium can be characterized by the first-period trading intensity of the low type for each asset and the buyer mixing probabilities conditional on $\pi_{i}(z)=\bar{\pi}$. Let $\gamma=\left\{\sigma_{A}, \sigma_{B}, \phi_{A}, \phi_{B}\right\}$ denote an arbitrary candidate equilibrium. In this section, we derive the set of $\gamma$ that constitute equilibria and therefore the set of all PBE.

Let us briefly outline how we will proceed. We start by analyzing a benchmark with uncorrelated assets in which information spillovers are not present. We show that the equilibrium of this benchmark is unique and features a strictly positive probability of trade in the first period (Proposition 1). We then reintroduce correlation, but take the trading behavior for asset $j$ as given and analyze the "partial equilibrium" for asset $i$. We show that, for each $\left(\sigma_{j}, \phi_{j}\right)$, there is a unique $\left(\sigma_{i}, \phi_{i}\right)$ that is consistent with a partial equilibrium for asset $i$, which may involve $\sigma_{i}=0$ (Proposition 2). A full equilibrium of the model is then simply a fixed point, i.e., $\left(\sigma_{i}, \phi_{i}\right)$ is consistent with an equilibrium for asset $i$ given $\left(\sigma_{j}, \phi_{j}\right)$ and vice versa. We argue that any fixed point must be symmetric and involves strictly positive probability of trade in the first period: $\sigma_{i}=\sigma_{j}>0$ (Proposition 3). We then characterize the set of fixed points and show that multiple equilibria arise when information spillovers are sufficiently strong (Theorem 1). Finally, we explore comparative statics with respect to the degree of asset correlation (Propositions 4 and 5).

\section{A. Benchmark without Information Spillovers}

In this section, we consider a benchmark in which information spillovers are absent because assets are uncorrelated (i.e., $\lambda=1-\pi$ ). From equations (5) and

\footnotetext{
${ }^{13}$ We adopt the convention that $\pi_{i}\left(b ; \sigma_{i}, 0\right)=\lim _{\sigma_{j} \rightarrow 0} \pi_{i}\left(b ; \sigma_{i}, \sigma_{j}\right)$. That is, buyers attribute an unexpected transaction to the low-type seller. This convention is without loss of generality.
} 
(6), the buyers' posterior belief $\pi_{i}$ is equal to the interim belief $\pi_{\sigma_{i}}$ defined in equation (4). Thus, there are no strategic interactions, and, by symmetry, it suffices to consider the equilibrium of a model with a single asset. The continuation value of a type- $\theta$ seller $i$ as it depends on the equilibrium trading strategies $\left(\sigma_{i}, \phi_{i}\right)$ is

$$
Q_{\theta}^{i}\left(\sigma_{i}, \phi_{i}\right)=(1-\delta) c_{\theta}+\delta F_{\theta}\left(\pi_{\sigma_{i}}, \phi_{i}\right)
$$

which is increasing in $\sigma_{i}$ and increasing in $\phi_{i}$ whenever $\pi_{\sigma_{i}}=\bar{\pi}$. For convenience, define $\bar{\sigma}>0$ such that $\pi_{\bar{\sigma}}=\bar{\pi}$. The following proposition characterizes the unique equilibrium of the benchmark.

PROPOSITION 1 (No Information Spillovers): If the assets are uncorrelated, then a unique equilibrium exists. In this equilibrium, $\sigma_{i}=\bar{\sigma}$ and $\phi_{i}$ is such that $Q_{L}^{i}\left(\bar{\sigma}, \phi_{i}\right)=v_{L}$

Thus, when there are no information spillovers, there is a unique equilibrium with positive trade. We will next show how these results change when asset values are correlated.

\section{B. Partial Equilibrium}

We now reintroduce positive correlation. As mentioned above, we start by taking the behavior of agents with respect to asset $j$ as given and define a partial equilibrium as follows.

DEFINITION 1: We say that $\left(\sigma_{i}, \phi_{i}\right)$ is a partial equilibrium for asset $i$ given $\left(\sigma_{j}, \phi_{j}\right)$ if buyers' beliefs about asset $i$ are updated according to (6) and the strategies induced by $\left(\sigma_{i}, \phi_{i}\right)$ satisfy buyer and seller optimality for asset $i .14$

Note that this definition does not require the behavior of seller $j$ to satisfy equilibrium conditions given $\left(\sigma_{i}, \phi_{i}\right)$, hence the "partial" moniker. To characterize partial equilibria, it will be useful to write the continuation value of a type- $\theta$ seller explicitly as it depends on the equilibrium strategies

$$
Q_{\theta}^{i}\left(\sigma_{i}, \sigma_{j}, \phi_{i}\right) \equiv(1-\delta) c_{\theta}+\delta \sum_{z \in\{b, g\}} \rho_{\theta}^{i}(z) F_{\theta}\left(\pi_{i}(z), \phi_{i}\right)
$$

Notice that seller $i$ 's expected continuation value is independent of $\phi_{j}$, but it depends crucially on $\sigma_{j}$ because seller $j$ 's trading strategy determines the distribution of news and hence the distribution over $\pi_{i}$.

LEMMA 4: Fix an arbitrary $\left(\sigma_{j}, \phi_{j}\right) \in[0,1]^{2}$. Then $\left(\sigma_{i}, \phi_{i}\right) \in[0,1]^{2}$ is a partial equilibrium for asset $i$ if and only if $Q_{L}^{i}\left(\sigma_{i}, \sigma_{j}, \phi_{i}\right) \geq v_{L}$, where the inequality must hold with equality if $\sigma_{i}>0$.

\footnotetext{
${ }^{14}$ For notational consistency we refer to a partial equilibrium as a pair $\left(\sigma_{i}, \phi_{i}\right)$; however, $\phi_{i}$ is not required to characterize equilibrium behavior if the posterior is never equal to $\bar{\pi}$.
} 
To understand the necessity of the inequality, suppose that $Q_{L}^{i}<v_{L}$. In this case, a low-type seller $i$ would strictly prefer to accept the bid in the first period and therefore seller optimality requires $\sigma_{i}=1$, which violates Lemma 3.

PROPOSITION 2 (Unique Partial Equilibrium): Fix an arbitrary $\left(\sigma_{j}, \phi_{j}\right) \in[0,1]^{2}$. There exists a unique $\sigma_{i}$ consistent with a partial equilibrium for asset $i$, which may involve $\sigma_{i}=0$, in which case seller $i$ simply "waits for news" (i.e., trades with probability zero). 15

That a partial equilibrium may involve $\sigma_{i}=0$ is similar to Daley and Green (2012), in which news is generated by an exogenous process. In their continuous-time setting with exogenous news, it is in fact necessary that equilibria involve periods of no trade. In our setting, news is endogenously generated by trade of another asset, which, as we will see, eliminates the possibility of a period with no trade once we solve simultaneously for a "full" equilibrium of the model.

Before doing so, consider the effect of $\sigma_{j}$ on $Q_{L}^{i}$. As $\sigma_{j}$ increases, there are two forces to consider. First, higher $\sigma_{j}$ makes good news more valuable; conditional on good news, buyers' posterior about asset $i$ is more favorable and hence the expected price is higher. Note that there is no analogous effect following bad news; conditional on $z_{i}=b$, buyers know that $\theta_{j}=L$ but their belief about seller $i$ (and hence the expected price) is independent of $\sigma_{j}$. The second effect is that higher $\sigma_{j}$ makes bad news more likely from the perspective of a low-type seller $i$. These two forces push in opposite directions and either one can dominate. Hence, $Q_{L}^{i}$ may increase or decrease with $\sigma_{j}$. The upshot is that, as seller $j$ trades more aggressively, the partial equilibrium for asset $i$ will involve seller $i$ trading more or less aggressively if the bad or good news effect, respectively, dominates.

To illustrate these two effects graphically, we plot $Q_{L}^{i}$ as a function of $\sigma_{i}$ for four different levels of $\sigma_{j}$ in Figure 2, panel A. Notice that moving from $\sigma_{j}=0$ to $\sigma_{j}=0.3$, seller $i$ must trade less aggressively in order to maintain $Q_{L}^{i}=v_{L}$. Further, when $\sigma_{j}=0.6, Q_{L}^{i}$ lies above $v_{L}$ everywhere and hence seller $i$ strictly prefers to wait. Finally, for $\sigma_{j}=0.9$, the bad news effect dominates and seller $i$ must trade more aggressively to maintain indifference.

As we show below, in general, the good news effect dominates initially (i.e., for low $\sigma_{j}$ ), while the bad news effect dominates eventually (i.e., for high $\sigma_{j}$ ). To formalize this result, define the mapping from seller $j$ 's trading strategy into the corresponding partial equilibrium trading strategy of seller $i$ by $S(\cdot)$, where $S\left(\sigma_{j}\right)=0$ if $Q_{L}^{i}\left(0, \sigma_{j}, 0\right) \geq v_{L}$ and $S$ satisfies $Q_{L}^{i}\left(S\left(\sigma_{j}\right), \sigma_{j}, \phi_{i}\right)=v_{L}$ for some $\phi_{i} \in[0,1]$ otherwise. Figure 2, panel $\mathrm{B}$, illustrates a plot of $S$, which is characterized in the following lemma.

LEMMA 5 (Strategic Interactions): Suppose that both $\delta$ and $\lambda$ are sufficiently large. Then there exist $\sigma_{1}<\sigma_{2} \in(0,1)$ such that:

(i) S is strictly decreasing on $\left[0, \sigma_{1}\right]$;

${ }^{15}$ Generically, the $\phi_{i}$ consistent with a partial equilibrium is also unique (see Proof of Proposition 2). 
Panel A. Continuation value

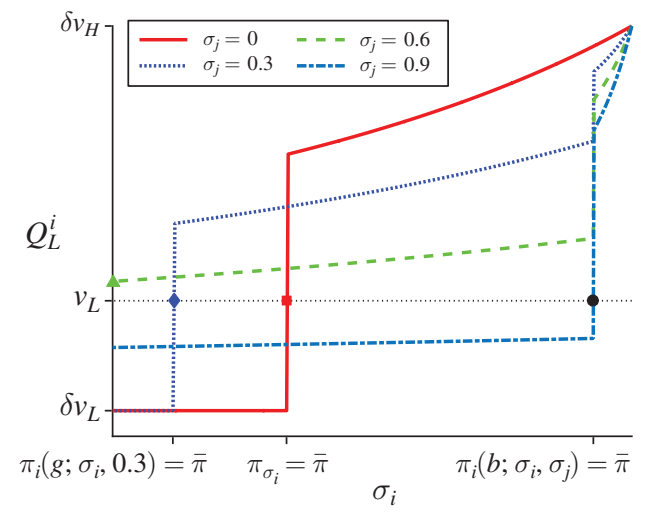

Panel B. Partial equilibrium

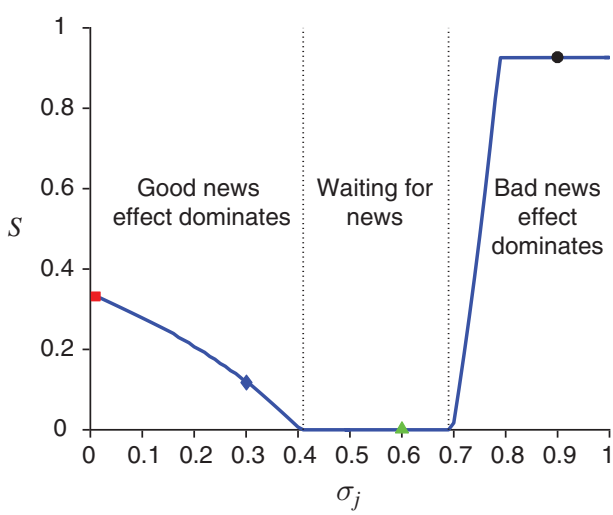

Figure 2. Partial Equilibria and Strategic Interactions

Notes: Panel A illustrates how the continuation value of a low-type seller $i$ depends on both $\sigma_{i}$ and $\sigma_{j}$. Panel B illustrates the set of partial equilibria for asset $i$ taking $\sigma_{j}$ as given. The four markers in the panel $\mathrm{B}$ correspond to the partial equilibria for the four different levels of $\sigma_{j}$ plotted in panel A. Parameters used: $\delta=0.7, \lambda=0.9$. The following parameters remain fixed throughout all figures: $c_{L}=0, c_{H} 0.2, v_{L}=0.1, v_{H}=0.25$.

(ii) $S(\sigma)=0$ for $\sigma \in\left[\sigma_{1}, \sigma_{2}\right]$;

(iii) $S$ is increasing on $\left[\sigma_{2}, 1\right]$.

To understand the intuition, suppose first that $\sigma_{j}=0$, in which case, just as in Proposition 1, the unique partial equilibrium involves $\sigma_{i}=\bar{\sigma}$ such that buyers' interim belief following a rejection in the first period is $\bar{\pi}$. Because there are no information spillovers from asset $j$, buyers' belief at $t=2$ is also $\bar{\pi}$. Hence, buyer mixing at $t=2$ is consistent with a partial equilibrium and $\phi_{i}$ is such that $Q_{L}^{i}\left(\bar{\sigma}, 0, \phi_{i}\right)=v_{L}$.

Next, consider an arbitrarily small $\sigma_{j}>0$ and suppose $\sigma_{i}$ remains fixed at $\bar{\sigma}$. Then, with probability arbitrarily close to one, seller $j$ does not trade at $t=1$, which is good news and leads to $\pi_{i}(g)>\pi_{\sigma_{i}}=\bar{\pi}$. By Lemma 1, the bid at $t=2$ must be $V\left(\pi_{i}(g)\right)>c_{H}$ with probability arbitrarily close to one. Thus, the good news effect causes a discontinuous increase in $Q_{L}^{i}$, whereas the bad news effect (with arbitrarily small probability, bad news arrives and the bid is $v_{L}$ ) is continuous. Clearly the good news effect dominates and in order to restore $Q_{L}^{i}=v_{L}, \sigma_{i}$ must decrease such that $\pi_{i}(g)=\bar{\pi}$. Hence $S$ is decreasing on $\left(0, \sigma_{1}\right)$, where $\sigma_{1}$ is such that $\pi_{i}\left(g, 0, \sigma_{1}\right)=\bar{\pi}$.

For $\sigma_{j} \in\left(\sigma_{1}, \sigma_{2}\right)$, seller $i$ gets a high enough price following good news and the likelihood of bad news remains small enough, that she strictly prefers to wait. That is, $Q_{L}^{i}>v_{L}$ for all $\sigma_{i}$ (as in the dashed line in Figure 2, panel A), and therefore the partial equilibrium requires $\sigma_{i}=0$.

For $\sigma_{j} \geq \sigma_{2}$, the probability of bad news is sufficiently large that the low type would be strictly worse off by simply waiting. Hence, she resumes trading with strictly positive probability. In Figure 2, panel B, she does so at first (i.e., where $S$ is strictly increasing) in order to boost the price following good news while the price 

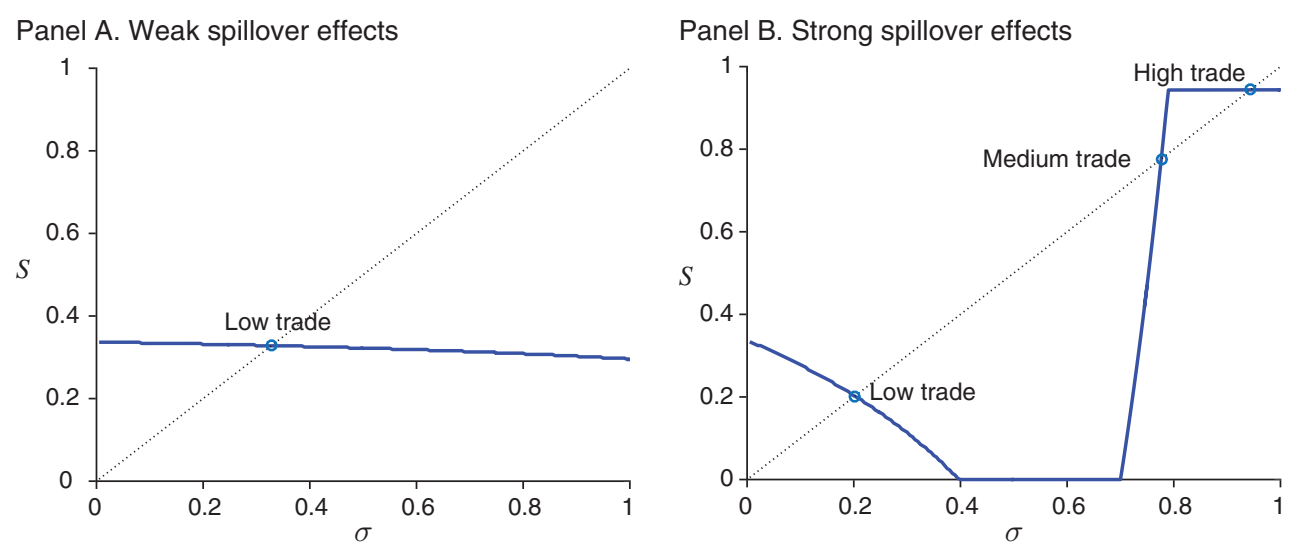

Figure 3. SPILlover EFFects AND Multiplicity

Notes: This figure illustrates the set of equilibria for two different levels of correlation. In panel A, asset values are only weakly correlated, whereas in panel B asset values are highly correlated.

following bad news remains $v_{L}$, but eventually (i.e., where $S$ is flat) the probability of bad news is so large that she trades in order to induce $\pi_{i}(b)=\bar{\pi}$ and her indifference is sustained through the buyer mixing probability after bad news. ${ }^{16}$

It is worth noting that the non-monotonicity in $S$ obtains when asset correlation is sufficiently large. When the correlation is low, the partial equilibrium involves $\pi_{i}(g)=\bar{\pi}$ and the good news effect dominates for all $\sigma_{j}$ (as in Figure 3 , panel A). Moreover, as the correlation goes to zero, $S$ converges uniformly to $\bar{\sigma}$.

\section{Full Equilibria}

Moving from a partial equilibrium for asset $i$ to an equilibrium across both assets requires that the trading strategies also satisfy $\sigma_{j}=S\left(\sigma_{i}\right)$ for $j \neq i$. That is, taken as given the offers for asset $i$ and the strategy of its seller, the offers for asset $j$ and the strategy of its seller must also correspond to a partial equilibrium. The following result shows that all equilibria must be symmetric and involve strictly positive probability of trade.

PROPOSITION 3 (Symmetry and News): In any equilibrium, $\sigma_{A}=\sigma_{B}>0$. If buyer mixing is part of an equilibrium, $\phi_{A}=\phi_{B}$.

Though symmetry is not an obvious property, the proof is actually quite simple. If $\sigma_{i}>\sigma_{j} \geq 0$ then $Q_{L}^{i}>Q_{L}^{j}$. But because $Q_{L}^{j}$ must be weakly bigger than $v_{L}$ (Lemma 4), the low-type seller $i$ strictly prefers to wait, which contradicts $\sigma_{i}>0$ satisfying seller optimality. The strict inequality in Proposition 3 then follows immediately: if $\sigma_{A}=\sigma_{B}=0$, then no news arrives and buyers' beliefs in the second period are exactly the same as in the first period, which would imply that $Q_{L}^{A}=Q_{L}^{B}<v_{L}$, again violating Lemma 4. Notice the contrast of this result

\footnotetext{
${ }^{16}$ Recall that $\pi_{i}(b)$ is independent of $\sigma_{j}$, which explains why $S$ is flat in this region.
} 
to the partial equilibrium (i.e., with exogenous news). When news is endogenously generated, it cannot be an equilibrium for either seller to simply wait for news.

Having established that any equilibrium must be symmetric, we drop the subscripts and denote an equilibrium by the pair $(\sigma, \phi)$. Furthermore, having established that any equilibrium involves $\sigma \in(0,1)$, the low type must be indifferent between accepting $v_{L}$ in the first period and waiting until the second period. Hence, any pair is an equilibrium if and only if

$$
Q_{L}(\sigma, \sigma, \phi)=v_{L}
$$

We have thus narrowed the search for equilibria to the solutions to equation (9). It is useful to note that potential equilibria can be classified into three different types depending on the posterior beliefs. Since the posterior beliefs are monotonic in the amount of trade in the first period, it is convenient to introduce the following definition.

DEFINITION 2: We define the three possible types of equilibria as

- Low trade: $\pi_{i}(g)=\bar{\pi}>\pi_{i}(b)$,

- Medium trade: $\pi_{i}(g)>\bar{\pi}>\pi_{i}(b)$, and

- High trade: $\pi_{i}(g)>\bar{\pi}=\pi_{i}(b) .17$

We denote the equilibrium trading intensity in the first period by $\sigma^{q}$ where $q \in\{$ low, med, high $\}$ and $\sigma^{\text {low }}<\sigma^{\text {med }}<\sigma^{\text {high }}$. Note that in a low (high) trade equilibrium, $\sigma^{q}$ is pinned down by $\pi_{i}\left(g, \sigma^{q}, \sigma^{q}\right)=\bar{\pi}\left(\pi_{i}\left(b, \sigma^{q}, \sigma^{q}\right)=\bar{\pi}\right)$ and $\phi^{q}$ is the solution to (9) given $\sigma^{q}$. In a medium trade equilibrium, buyer mixing is not part of the equilibrium (hence $\phi$ is undetermined) and $\sigma$ must solve (9). Using these observations, we now establish the main result of the paper.

THEOREM 1 (Characterization and Multiplicity): An equilibrium exists and there are at most three. More precisely, equilibria are characterized as follows:

(i) Low Trade.-There is at most one low trade equilibrium. There exists a $\bar{\delta}<1$, such that this equilibrium exists if $\delta>\bar{\delta}$.

(ii) High Trade.-There is at most one high trade equilibrium. Given $\delta$, there exist $\bar{\lambda}_{\delta}<1$ such that this equilibrium exists if $\lambda>\bar{\lambda}_{\delta}$.

(iii) Medium Trade.-There are at most two medium trade equilibria. Exactly one such equilibrium exists if $\delta>\bar{\delta}$ and $\lambda>\bar{\lambda}_{\delta}$.

The three types of equilibria coexist when $\delta>\bar{\delta}$ and $\lambda>\bar{\lambda}_{\delta}$. If correlation is sufficiently small, the equilibrium is generically unique and converges to the one in Proposition 1 as correlation goes to zero.

\footnotetext{
${ }^{17}$ The other two possible orderings of the posteriors $\pi_{i}(g)>\pi_{i}(b)>\bar{\pi}$ and $\bar{\pi}>\pi_{i}(g)>\pi_{i}(b)$ are ruled out by Lemma 1 and Assumption 2, respectively.
} 
The key insights of the theorem are illustrated in Figure 3, which considers two different levels of correlation. In panel B, correlation is relatively high, leading to strong spillover effects and three equilibria. ${ }^{18}$ In panel A, correlation is relatively low. Hence, the spillover effects are modest, which leads to a unique equilibrium with low trade.

\section{The Effects of Asset Correlation}

As we have just seen, asset correlation can lead to information spillovers and generate multiplicity. The level of correlation also has important implications for trading volume and welfare, which we now elaborate upon.

PROPOSITION 4 (Trading Volume): First-period trading volume increases with $\lambda$ in the high trade equilibrium, decreases with $\lambda$ in the low trade equilibrium, and can decrease with $\lambda$ in the medium trade equilibria.

Figure 4, panel A illustrates the effects of correlation on trading volume. Holding $\sigma$ fixed, increasing $\lambda$ makes bad news more likely from the low type's perspective (akin to the bad news effect) but increases the posterior belief following good news (akin to the good news effect). In the low trade equilibrium, $\pi_{i}(g)=\bar{\pi}$, hence $\sigma_{i}$ must decrease to offset the good news effect. In the high trade equilibrium, $\pi_{i}(b)=\bar{\pi}$, and since an increase in $\lambda$ leads to a lower belief following bad news, $\sigma_{i}$ must increase. In the medium trade equilibrium, both the good and bad news effects are at play in determining first-period trading volume. As in Figure 4, panel A, our numerical examples suggest that the good news effect dominates in the medium trade equilibrium and thus $\sigma$ decreases with $\lambda$, though we do not have a general proof of this result.

To understand the welfare implications, observe that because buyers are identical and competitive, they extract zero (expected) surplus. Next, recall that in any equilibrium the low-type sellers are indifferent between trading in the first period at a price of $v_{L}$ or waiting and trading in the second period. Hence, their ex ante equilibrium payoff is $v_{L}$ regardless of equilibrium or level of asset correlation. In order to study the welfare implications, it is therefore sufficient to consider the equilibrium payoff of a high-type seller, which we denote by $Q_{H}^{q}$, where $q \in\{l o w$, med, high $\}$ denotes the equilibrium. Moreover, any welfare improvement for the high-type seller is a Pareto improvement.

PROPOSITION 5 (Welfare): The following statements hold:

(i) Whenever the three equilibria coexist, the high trade equilibrium Pareto dominates the medium trade equilibrium, which Pareto dominates the low trade equilibrium (i.e., $Q_{H}^{\text {high }}>Q_{H}^{\text {med }}>Q_{H}^{\text {low }}$ ).

\footnotetext{
${ }^{18}$ Belief-based refinements (e.g., Intuitive Criterion or Divinity) do not eliminate any of the equilibria since there are no off-path events. However, it is worth noting that the medium trade equilibrium is less "stable" than the other two equilibria in the following sense. When all three equilibria coexist, the function $S\left(\sigma_{j}\right)$ crosses the 45-degree line from below at $\sigma^{\text {med }}$ (see Figure 3, panel B). Thus, if players started from a conjecture that $\sigma_{j}$ was just above or below $\sigma^{\text {med }}$, the partial equilibrium for asset $i$ would be even further away from $\sigma^{\text {med }}$.
} 
Panel A. Volume

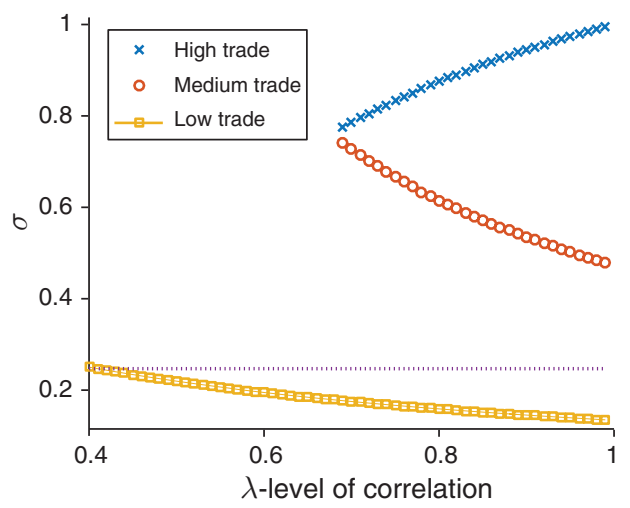

Panel B. Welfare

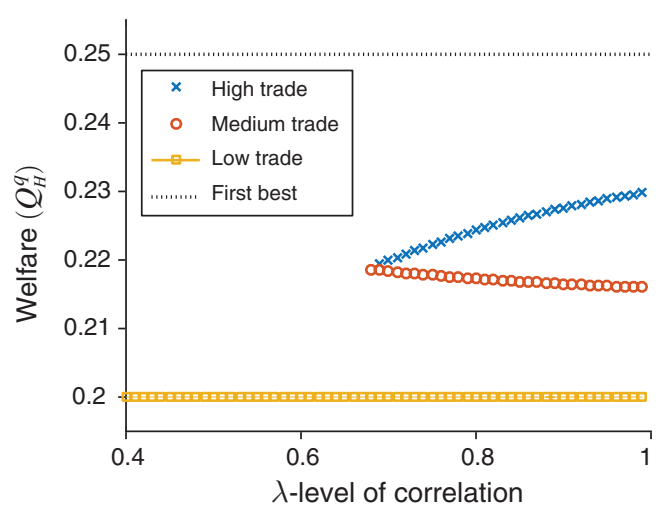

Figure 4. The EfFect of Correlation

Notes: Panel A illustrates the set of equilibrium $\sigma$ as they depend on the level of correlation and the dotted black line is the unique equilibrium $\sigma$ when asset values are uncorrelated. Panel B illustrates welfare as it depends on the level of correlation.

(ii) $Q_{H}^{\text {high }}$ increases with $\lambda, Q_{H}^{\text {med }}$ can decrease with $\lambda$, and $Q_{H}^{\text {low }}=c_{H}$ for all $\lambda$.

The first part of the proposition offers a welfare ranking across equilibria. In the low trade equilibrium, following both good and bad news, the high-type seller's equilibrium payoff is $c_{H}$. In both the medium and high trade equilibria, after good news the buyers' beliefs satisfy $\pi_{i}(g)>\bar{\pi}$. Hence, after good news the price offered is strictly above $c_{H}$ and after bad news the high type is no worse off. This immediately implies that in both of these equilibria, the high-type seller is strictly better off than in the low trade equilibrium. In fact, the high-type seller's payoff is strictly increasing in $\sigma$ for all $\sigma \geq \sigma^{l o w}$ : more aggressive trading by the low type in the first period leads to less adverse selection, higher prices and more trade in the second period. Therefore, the high trade equilibrium Pareto dominates the medium trade one.

Holding $\sigma^{q}$ fixed, $Q_{H}^{q}$ increases with $\lambda$. Thus, it is natural to conjecture that welfare improves with the level of correlation. However, the second part of the proposition shows that the welfare comparatives statics are considerably more nuanced. In particular, $\sigma^{\text {low }}$ decreases with $\lambda$ at a rate that precisely offsets any welfare gains so that welfare remains independent of the degree of correlation in the low trade equilibrium. Further, $\sigma^{\text {med }}$ can decrease sufficiently fast that welfare is actually decreasing in $\lambda$ in the medium trade equilibrium. On the other hand, $\sigma^{\text {high }}$ increases with $\lambda$ such that the gains of increasing correlation are amplified in the high trade equilibrium.

Figure 4, panel B, offers a graphical illustration of Proposition 5. When correlation is low, there is a unique equilibrium, which involves low trade. In this equilibrium, the high type is indifferent whether to trade or not following good news and any increase in correlation results in a decrease in $\sigma$ keeping total welfare unchanged. When correlation becomes sufficiently large, information spillovers become relevant and multiplicity kicks in. Higher correlation reduces welfare along the medium trade equilibrium because $\sigma^{\text {med }}$ decreases sufficiently fast (see Figure 4, panel A), which leads to less efficient trade in the first period and more adverse selection in the second period. On the other hand, in the high trade equilibrium, the 
low types trade more aggressively as correlation increases, leading to less adverse selection in the second period and higher welfare.

\section{Post-Trade Transparency in Opaque Markets}

The extent to which information spillovers can impact market behavior depends crucially on the ability of agents to observe previous transactions. Up to this point, we have assumed that all trades take place in one market and that all potential buyers observe all previous transactions. In practice, trades often take place with less transparency. Not all participants observe all assets for sale nor do they necessarily have access to a database of prior transactions.

The corporate bond market prior to the introduction of TRACE is a good example of such a setting. Bessembinder and Maxwell (2008) describe the pre-TRACE corporate bond market as an "opaque environment" in which completed transactions “were not made public." Beginning in July 2002, regulators required bond dealers to report all trades in publicly issued corporate bonds to the National Association of Security Dealers, which in turn made transaction data available to the public.

Changes in the degree of transparency can also materialize through market-based mechanisms. For instance, as discussed earlier, recent innovations in information technologies and the introduction of websites such as Zillow, RedFin, and Trulia have greatly increased the post-trade transparency in real estate markets.

In this section, we use our framework to explore the implications of introducing post-trade transparency into an otherwise opaque market. To do so, we modify the model slightly so that not all buyers necessarily observe all transactions. Specifically, we assume that each asset trades on a different "platform" and potential buyers only observe transactions on the platform in which they participate. We will first analyze the case in which buyers are symmetric: each buyer participates on only one platform. Buyers on platform $i$ observe transactions of asset $i$ but not asset $j$ and vice versa for buyers on platform $j$. We then extend our analysis to a setting in which one buyer participates on both platforms and has superior information to other potential buyers.

\section{A. Symmetric Buyers}

Absent post-trade transparency, buyers on platform $i$ do not know whether asset $j$ sold. In this case, we say the market is opaque. In an opaque market, information spillovers are not possible and, regardless of the true underlying correlation, the equilibrium looks $a s$ if asset values are uncorrelated (Proposition 1).

In the presence of post-trade transparency, a transaction in the first period becomes public information across all buyers in the second period and the set of equilibria depend on the level of correlation as in Theorem 1. Thus, post-trade transparency facilitates information spillovers in markets that are otherwise opaque, which can lead to multiple equilibria and have consequences for both trade volume and welfare.

PROPOSITION 6 (Effect of Post-Trade Transparency): If the market is opaque then, regardless of $\lambda$, there are no information spillovers and a unique equilibrium 
exists (as in Proposition 1). Suppose that $\delta>\bar{\delta}$ as in Theorem 1; then introducing post-trade transparency has the following implications:

(i) Multiple equilibria will exist if $\lambda$ is sufficiently high. Depending on which equilibrium is played, trade volume may increase or decrease while welfare weakly increases.

(ii) The equilibrium remains unique if $\lambda$ is not sufficiently high. Trade volume will decrease while welfare remains unchanged.

Indeed, there is mixed empirical evidence as to the effects of TRACE. Asquith, Covert, and Pathak (2013) find that it led to a significant decline in trading activity for high-yield bonds. This is in contrast to a controlled study by Goldstein, Hotchkiss, and Sirri (2007), who find no conclusive evidence that TRACE caused a reduction in trading activity. Proposition 6 helps to reconcile these findings: introducing post-trade transparency can increase or decrease trading activity depending on the correlation and which equilibrium is played.

In the online Appendix, we extend our results to intermediate levels of post-trade transparency. In particular, we posit that buyers on platform $i$ observe a transaction on platform $j$ with some intermediate probability $\xi \in(0,1)$. Fixing $\delta>\bar{\delta}$ and $\lambda>\bar{\lambda}_{\delta}$ as in Theorem 1, we show that there exists a critical level of posttrade transparency $\bar{\xi} \in(0,1)$ such that the three equilibria in Definition 2 coexist if $\xi>\bar{\xi}$. Whereas the equilibrium is unique when the level of post-trade transparency (as measured by $\xi$ ) is sufficiently small.

We then analyze the welfare implications in more detail by conducting comparative statics on $\xi$. Some post-trade transparency $(\xi>0)$ always weakly increases welfare relative to an opaque market $(\xi=0)$. Moreover, both volume and welfare are increasing in $\xi$ in the high trade equilibrium. However, starting from $\xi \in(0,1)$, increasing post-trade transparency is not guaranteed to improve the outcome. For instance, as $\xi$ increases, both volume and welfare decrease in the medium trade equilibrium. In the low trade equilibrium, volume decreases with $\xi$ while welfare remains unchanged. These results further emphasize the insight that publishing data about trading activity can alter its information content and potentially lead to worse outcomes.

\section{B. Asymmetric Buyers}

Pro-transparency policies are sometimes motivated as a way to "level the playing field" between traders with different levels of access to information. In this section, we evaluate the effects of such policies in a setting where one buyer has better access to transaction data than others. For example, broker-dealers are active in many markets, which gives them an informational advantage over investors who participate less frequently or in fewer markets.

To capture this feature, we now assume that one of the buyers is active on both trading platforms and is therefore able to observe the history of transactions of both assets regardless of whether there is post-trade transparency. For convenience, we refer to this buyer as an insider. The remaining buyers, whom we refer to as 
investors, participate on either platform $i$ or $j$, but not both. ${ }^{19}$ In this section, we will also assume that in each period the seller holds a second price auction with a secret reserve price. Adopting this trading mechanism (rather than Bertrand competition) is primarily to simplify the equilibrium analysis and intuition. ${ }^{20}$

Remark 2: When post-trade transparency is in place, investors have the same information as the insider and the set of equilibrium is the same as in Theorem 1. Therefore, both investors and the insider make zero (expected) trading profits.

When the market is opaque, investors' degree of sophistication is an important determinant of whether they benefit from a policy inducing post-trade transparency. Below, we consider two different specifications regarding the degree of sophistication of investors. They can be either "naïve" or "sophisticated" regarding the fact that they are facing competition from a trader with access to better information. One can think of naïve investors as utility maximizing agents with an incorrect prior belief about the probability that an insider is present (i.e., the true probability is one but naïve investors believe it is zero). Sophisticated investors are fully rational agents.

Regardless of investors' sophistication, several familiar features arise in equilibrium. The bid in the first period is $v_{L}$. The first period bid is rejected by the high-type seller with probability one and accepted by the low-type seller with probability $\sigma_{i}$. We characterize equilibrium behavior in the second period separately for each case (naïve/sophisticated investors) below.

Naïve Investors. - If the market is opaque then, in the second period, naïve investors bid according to Lemma 1, where their posteriors are conditioned only on $\sigma_{i}$. The insider bids the unconditional expected value if her posterior is above $\bar{\pi}$ and $v_{L}$ otherwise. Naïve investors fall prey to a winner's curse. They win the auction only when the insider receives bad news from the other platform. Conditional on asset $j$ trading in the first period, naïve investors' bid underestimates the probability of asset $i$ being low value and hence, on average, they experience trading losses. On the other hand, when asset $j$ does not trade, then naïve investors are always outbid by the insider who is thus able to capture information rents. Also, since the second highest bid always originates from investors, the seller faces exactly the distribution of bids as if he were facing only naïve investors. This implies that any rents made by the insider are exactly offset by the losses of the investors. Thus, in addition to the potential for multiple equilibria and welfare gains for the seller, post-trade transparency has a redistributive effect from the insider to the naïve investors.

PROPOSITION 7 (Naïve Investors): If investors are naïve and the market is opaque, there exists a unique equilibrium. This equilibrium generates the same total surplus

\footnotetext{
${ }^{19}$ Our results extend to the case in which an insider is present with probability $\epsilon \in(0,1)$. The results can also be extended to a setting with multiple insiders as long as they are able to capture some information rents (e.g., the insiders are not identically informed). If multiple insiders compete away all information rents, the analysis of Section III applies.

${ }^{20}$ When buyers are symmetric (or there is public reporting), both trading mechanisms lead to exactly the same equilibrium outcomes. In an opaque market with asymmetric buyers and Bertrand competition, the equilibrium construction is more complex because the optimal bidding strategy depends on the distribution of other bids, which can require that buyers mix over a continuum of offers. Nevertheless, the key insights in Propositions 7 and 8 are robust to Bertrand competition (formal results available upon request).
} 
as the low trade equilibrium in Theorem 1. However, the insider makes positive trading profits while naïve investors experience trading losses. Therefore, introducing post-trade transparency reduces insider trading profits and increases the welfare of naïve investors.

Sophisticated Investors. - When investors understand that they are competing against an insider, they are aware of the winner's curse. Therefore, when the market is opaque, sophisticated investors bid in the second period as if the asset on the other platform traded in the first period. Note that a bid of $v_{L}$ w.p. 1 in the second period cannot be part of an equilibrium. Therefore, it must be that $\pi_{i}(b)=\bar{\pi}$, which requires a higher equilibrium $\sigma$ than if all buyers are symmetric. ${ }^{21}$ The increased probability of trade in the first period enhances efficiency relative to the symmetric buyer case. Yet, all the additional surplus goes to the insider and not the seller who still faces the same distribution of offers in the second period. On the other hand, with post-trade transparency, the insider faces competition from sophisticated investors and thus loses his rents. Unlike with naïve buyers, the reduction in the insider's rents is not purely redistributive.

PROPOSITION 8 (Sophisticated Investors): When investors are sophisticated and the market is opaque, there exists a unique equilibrium that Pareto dominates the low trade equilibrium in Theorem 1. The additional surplus is captured entirely by the insider. Therefore, introducing post-trade transparency reduces insider profits without affecting sophisticated investors' welfare but may decrease overall trading surplus.

Importantly, in both cases, insiders prefer opacity. This may help explain why insiders in financial markets lobbied against rapid dissemination of transaction data to all market participants. ${ }^{22}$ In addition, our results show that although post-trade transparency can help protect naïve investors, there is a risk of reducing welfare if applied in an environment where investors are sophisticated.

It is worth noting the contrast to the case with symmetric buyers, in which posttrade transparency cannot lead to lower welfare. The difference in the results stems from the fact that when there is an insider who is better informed, there is effectively less competition in the second period. This makes the seller more pessimistic about the future and thus increases her willingness to trade early. Thereby, opacity has the potential to mitigate the seller's incentive to delay trade and increase welfare provided that investors are sophisticated.

\section{Concluding Remarks}

We study the effects of information spillovers in a setting where asset values are correlated and sellers have private information. We highlight novel feedback effects

\footnotetext{
${ }^{21}$ Here, $\pi_{i}(b)$ can be interpreted either as the belief of the insider conditional on observing a trade of asset $j$ or the belief of investors on platform $i$ reasoning about asset $i$ as if asset $j$ traded.

${ }^{22}$ In a letter to the SEC, the Bond Market Association, which represents bond dealers and underwriters, stated that its membership had "serious concerns about potential harm to liquidity resulting from rapid dissemination of transaction data on lower rated, less frequently traded issues." Note that these are precisely the bonds where information asymmetries are likely to be most relevant (see www.sifma.org/issues/item.aspx?id=779).
} 
between the information content in markets and the incentive to trade in a dynamic setting. The endogenous nature of information introduces strategic interactions in the trading behavior of sellers. When correlation is sufficiently high, we show that these interactions lead to multiple equilibria that differ in their trading volume, prices, information content, and welfare.

Information spillovers require that transactions be observable to market participants. When markets are opaque, the equilibrium is unique. Hence, introducing posttrade transparency can lead to multiplicity, which can increase or decrease trading activity depending on the level of correlation and which equilibrium is played. This finding has implications for how we interpret empirical work. For instance, it can help explain why Bessembinder, Maxwell, and Venkataraman (2006) and Edwards, Harris, and Piwowar (2007) find that market participants gain from the introduction of TRACE, while Goldstein, Hotchkiss, and Sirri (2007) see no effect within a subclass of securities, and Asquith, Covert, and Pathak (2013) find significantly different results for bonds that were part of the different phases of the TRACE program.

Our findings are also relevant from a policy perspective. In order to achieve potential welfare gains associated with post-trade transparency, it is important to steer market participants to coordinate on the correct equilibrium. When market participants have differential access to transaction data, post-trade transparency indeed "levels the playing field" if investors are naïve; it reduces both insiders' trading profits and investors' trading losses. However, post-trade transparency does not benefit sophisticated investors and can potentially reduce total surplus. Therefore, it is important to take into account the type of market participants when considering such policies.

Another relevant pro-transparency policy is the introduction of published benchmarks (e.g., LIBOR). Duffie, Dworczak, and Zhu (forthcoming) analyze the role of benchmarks in revealing information about fundamentals. Their paper suggests that the introduction of benchmarks is welfare enhancing. In our setting, the history of transactions can also reveal information about fundamentals. Our analysis highlights an important consideration absent in their setting, which is that the informational content of the benchmark may change once it is published. Due to feedback effects, publishing benchmarks may reveal more or less information than expected and can have additional consequences for trading behavior. Thus, when considering an introduction of a benchmark, it is important to understand the extent to which its information content is endogenous.

\section{APPENDIX}

\section{PROOF OF LEMMA 1:}

For $(i)$ and $(i i)$, see the proof of Lemma 1 in Daley and Green (2016). ${ }^{23}$ Moreover, by their Lemma A.3, the bid price must earn zero expected profit. To demonstrate (iii), we will show that the bid price must be either $v_{L}$ or $c_{H}$ when $\pi_{i}=\bar{\pi}$ by ruling out all other bids.

\footnotetext{
${ }^{23}$ Conditional on reaching the second trading period and the buyer's belief, $\pi_{i}$, the strategic setting for a single asset $i$ is identical to theirs.
} 
Clearly, at $t=2$, the reservation price of the low-type seller is $c_{L}$ and the reservation price of the high-type seller is $c_{H}$. Hence, if the bid is strictly above $c_{H}$, both types will accept w.p.1 and the winning buyer earns negative expected profit. Next, suppose there is positive probability that the bid is strictly less than $v_{L}$. Then, for $\epsilon>0$ small enough, a buyer could earn strictly positive expected profit by deviating and offering $v_{L}-\epsilon$. Finally, if the bid is strictly between $v_{L}$ and $c_{H}$, the high type will reject, the low type will accept, and the winning buyer makes negative profit. Thus, we have shown that the equilibrium bid price at $t=2$ when $\pi_{i}=\bar{\pi}$ must be either $v_{L}$ or $c_{H}$.

\section{PROOF OF LEMMA 2:}

Since $c_{H}>c_{L}$ and $F_{H} \geq F_{L}$, we have that

$$
Q_{L}^{i}=(1-\delta) \cdot c_{L}+\delta \cdot E_{L}\left\{F_{L}\left(\pi_{i}, \phi_{i}\right)\right\} \leq(1-\delta) \cdot c_{H}+\delta \cdot E_{L}\left\{F_{H}\left(\pi_{i}, \phi_{i}\right)\right\} .
$$

Therefore, it is sufficient to show that $E_{H}\left\{F_{H}\left(\pi_{i}, \phi_{i}\right)\right\} \geq E_{L}\left\{F_{H}\left(\pi_{i}, \phi_{i}\right)\right\}$. Recall that $F_{H}$ is increasing in $\pi_{i}$ and independent of $\phi_{i}$. Hence, the desired inequality is implied by proving that conditional on $\theta_{i}=H$, the random variable $\pi_{i}$ (weakly) first-order stochastically dominates $\pi_{i}$ conditional on $\theta_{i}=L$.

Note that the distribution of $\pi_{i}$ in the second period is a function of the trading probabilities by both types and the realization of news from the other seller, $z_{i} \in\{$ trade, notrade $\}$. For $\theta \in\{L, H\}$ and $z \in\{$ trade, notrade $\}$, define $\rho_{\theta}(z)$ $\equiv \operatorname{Pr}\left(z_{i}=z \mid \theta_{i}=\theta\right)$. Fix the interim belief $\pi_{i}^{\text {Int }}$, and for $z^{\prime}, z^{\prime \prime} \in\{$ trade, notrade $\}$ with $z^{\prime} \neq z^{\prime \prime}$, say that $z_{i}=z^{\prime}$ is "good news" if the posterior $\pi_{i}$ satisfies $\pi_{i}\left(z^{\prime}\right) \geq \pi_{i}\left(z^{\prime \prime}\right)$, and it is "bad news" otherwise. Without loss of generality, suppose that $z^{\prime}$ is good news and $z^{\prime \prime}$ is bad news. But note that this implies

$$
\begin{aligned}
& \frac{\pi_{i}^{\text {Int }} \cdot \rho_{H}\left(z^{\prime}\right)}{\pi_{i}^{\text {Int }} \cdot \rho_{H}\left(z^{\prime}\right)+\left(1-\pi_{i}^{\text {Int }}\right) \cdot \rho_{L}\left(z^{\prime}\right)}=\pi_{i}\left(z^{\prime}\right) \geq \pi_{i}\left(z^{\prime \prime}\right) \\
& =\frac{\pi_{i}^{I n t} \cdot \rho_{H}\left(z^{\prime \prime}\right)}{\pi_{i}^{\text {Int }} \cdot \rho_{H}\left(z^{\prime \prime}\right)+\left(1-\pi_{i}^{\text {Int }}\right) \cdot \rho_{L}\left(z^{\prime \prime}\right)}
\end{aligned}
$$

with $\pi_{i}(z) \equiv \pi_{i}^{\text {Int }}$ if $\rho_{H}(z)=\rho_{L}(z)=0$. Combining with the fact that $\rho_{\theta}\left(z^{\prime \prime}\right)=1-\rho_{\theta}\left(z^{\prime}\right)$ for $\theta=L, H$, we have that $\rho_{H}\left(z^{\prime}\right) \geq \rho_{L}\left(z^{\prime}\right)$, which establishes the result.

\section{PROOF OF LEMMA 3:}

(i) From Lemma 2, the strict ranking of seller continuation values implies that, in any equilibrium, if the high type is willing to accept an offer with positive probability then the low type must accept w.p.1. Thus, given Assumption 1, any bid at or above $c_{H}$ would lead to negative expected profit. Any bid in $\left(v_{L}, c_{H}\right)$ also leads to losses since it is only accepted by the low type. If the bid was strictly less than $v_{L}$, a buyer can make strictly positive profits by offering $v_{L}-\epsilon$, for $\epsilon>0$ small enough. Thus, any deterministic offer strictly below $v_{L}$ can be ruled out. The only deterministic bid possible is $v_{L}$, at this 
point there is no profitable deviation for the other buyer than offering $v_{L}$ as well. The same arguments rule out any mixed strategy equilibrium that has a mass point anywhere other than $v_{L}$. Finally, mixing continuously over some interval of offers cannot be an equilibrium. We show this by contradiction. If one of the buyers mixes over some interval $[\underline{b}, \bar{b}]$ with $\bar{b}=v_{L}$ then the other buyer must be offering $v_{L}$ with probability 1 because otherwise he would never want to offer $v_{L}$, which leads to zero profits w.p.1. If instead $\bar{b}<v_{L}$, the other buyer's best response can never have $\underline{b}$ (or anything below) as part of its support. This bid will lose with probability 1 and thus earn zero profits, while bidding $\frac{\bar{b}+v_{L}}{2}$ would lead to strictly positive profits.

(ii) Clearly the high type would reject $v_{L}$, since $v_{L}<c_{H}$. To see that the low type must accept with probability less than one, note that if in equilibrium the low type accepted w.p.1, then the posterior belief would assign probability 1 to the type being high in the next period. The offer in the second period (as argued in Lemma 1) would then be $v_{H}$ but, given Assumption 2, the low-type seller prefers to deviate and trade in period 2 at $v_{H}$ rather than trade at $v_{L}$ in period 1.

\section{PROOF OF PROPOSITION 1:}

Let $\bar{\sigma}$ be defined by $\pi_{\sigma_{i}}=\bar{\pi}$, which is unique and by Assumption 1 satisfies $\bar{\sigma} \in(0,1)$. Suppose an equilibrium exists and has $\sigma_{i}<\bar{\sigma}$ (if and only if $\left.\pi_{\sigma_{i}}<\bar{\pi}\right)$. Then by Lemma 1 we have $Q_{L}^{i}=(1-\delta) c_{L}+\delta v_{L}<v_{L}$, which contradicts $\sigma_{i}<1$. Suppose instead that an equilibrium exists and has $\sigma_{i}>\bar{\sigma}$ (if and only if $\pi_{\sigma_{i}}>\bar{\pi}$ ). Then by Lemma 1 and Assumption 2 we have $Q_{L}^{i}=(1-\delta) c_{L}+\delta V\left(\pi_{\sigma_{i}}\right)>(1-\delta) c_{L}+\delta c_{H}>v_{L}$, which contradicts $\sigma_{i}>0$. Hence, in any equilibrium, $\sigma_{i}=\bar{\sigma}$ (if and only if $\pi_{\sigma_{i}}=\bar{\pi}$ ), and the low type must be indifferent between trade at $t=1$ and trade at $t=2$, i.e., $v_{L}=Q_{L}^{i}\left(\bar{\sigma}, \phi_{i}\right)$. But note that $Q_{L}^{i}(\bar{\sigma}, \cdot)$ is monotonically increasing and continuous, with $Q_{L}^{i}(\bar{\sigma}, 0)=(1-\delta) c_{L}+\delta v_{L}<v_{L}$ and $Q_{L}^{i}(\bar{\sigma}, 1)=(1-\delta) c_{L}+\delta c_{H}>v_{L}$, which implies that $\phi_{i} \in(0,1)$ and that an equilibrium exists, is unique, with strategies $\left(\sigma_{i}, \phi_{i}\right)$ satisfying the stated conditions.

\section{PROOF OF LEMMA 4:}

See text for the proof of necessity. Sufficiency follows from Lemmas 1-3.

\section{PROOF OF PROPOSITION 2:}

Fix any $\left(\sigma_{j}, \phi_{j}\right) \in[0,1]^{2}$. We separate the analysis into two cases depending on whether $Q_{L}^{i}\left(0, \sigma_{j}, 1\right)$ is greater than $v_{L}$. First, suppose that $Q_{L}^{i}\left(0, \sigma_{j}, 1\right) \geq v_{L}$, then $\sigma_{i}=0$ satisfies the partial equilibrium as established in Lemma 4. Moreover, we claim that any $\sigma_{i}>0$ cannot be part of a partial equilibrium. To see why, note that $Q_{L}^{i}\left(0, \sigma_{j}, 1\right) \geq v_{L}$ implies that $\pi_{i}(g) \geq \bar{\pi}$ for any $\sigma_{i}>0$. In this region, $Q_{L}^{i}\left(\sigma_{i}, \sigma_{j}, 1\right)$ is strictly increasing in $\sigma_{i}$, which means that $Q_{L}^{i}\left(\sigma_{i}, \sigma_{j}, 1\right)>v_{L}$ for all $\sigma_{i}>0$, violating Lemma 4. Hence, there is no other candidate value for $\sigma_{i}$ satisfying the partial equilibrium as established in Lemma 4. Note that in this case the posteriors are generically not equal to $\bar{\pi}$; thus buyer mixing is not part of a partial equilibrium and $\phi_{i}$ is not required to characterize equilibrium behavior. 
Next, suppose that $Q_{L}^{i}\left(0, \sigma_{j}, 1\right)<v_{L}$, and note that $Q_{L}^{i}\left(1, \sigma_{j}, \phi_{i}\right)>v_{L}$ for any $\phi_{i} \in[0,1]$ by Assumption 2. Since $Q_{L}^{i}\left(\sigma_{i}, \sigma_{j},{ }^{\cdot}\right)$ is upper hemicontinuous in $\sigma_{i}$, there exists a unique $\sigma_{i}>0$ such that $Q_{L}^{i}\left(\sigma_{i}, \sigma_{j}, \phi_{i}\right)=v_{L}$ for some $\phi_{i} \in[0,1]$. Furthermore, such a $\sigma_{i}$ is unique since $Q_{L}^{i}$ is strictly increasing in $\sigma_{i}$ when $\pi_{i}(g) \geq \bar{\pi}$, which is the only relevant region. The uniqueness of $\phi_{i}$ follows from the fact that $Q_{L}^{i}$ is strictly increasing in $\phi_{i}$ when buyer mixing is part of the partial equilibrium.

The following technical result will be used in several subsequent proofs.

LEMMA A.1: For a given $\sigma_{i},(i) Q_{L}^{i}\left(\sigma_{i}, \sigma_{j}, 1\right)$ is strictly decreasing in $\sigma_{j}$ whenever $\pi_{i}(b)<\bar{\pi} \leq \pi_{i}(g)$, and (ii) $Q_{H}^{i}\left(\sigma_{i}, \sigma_{j}, \cdot\right)$ is strictly increasing in $\sigma_{j}$ whenever $\pi_{i}(b) \leq \bar{\pi} \leq \pi_{i}(g)$.

\section{PROOF:}

For $(i)$, when posteriors satisfy $\pi_{i}(b)<\bar{\pi} \leq \pi_{i}(g)$, we have

$$
Q_{L}^{i}\left(\sigma_{i}, \sigma_{j}, 1\right)=(1-\delta) c_{L}+\delta\left(\rho_{L}^{i}(b) v_{L}+\left(1-\rho_{L}^{i}(b)\right) V\left(\pi_{i}(g)\right)\right) .
$$

We can rewrite this expression as

$$
\begin{gathered}
\delta^{-1}\left(Q_{L}^{i}\left(\sigma_{i}, \sigma_{j}, 1\right)-(1-\delta) c_{L}\right)=\underbrace{\rho_{\text {unc }}^{i}(b) V\left(\pi_{i}(b)\right)+\left(1-\rho_{\text {unc }}^{i}(b)\right) V\left(\pi_{i}(g)\right)}_{\text {unconditional expected value }} \\
+\underbrace{\left(\rho_{L}^{i}(b)-\rho_{\text {unc }}^{i}(b)\right)}_{+} \underbrace{\left(V\left(\pi_{i}(b)\right)-V\left(\pi_{i}(g)\right)\right)}_{-}+\rho_{L}^{i}(b) \underbrace{\left(v_{L}-V\left(\pi_{i}(b)\right)\right)}_{-},
\end{gathered}
$$

where $\rho_{\text {unc }}^{i}(b)=\sigma_{j}(1-\pi)$ is the unconditional probability of bad news. The first term on the RHS is the unconditional expected value of the asset in $t=2$, which is independent of $\sigma_{j}$ by the law of iterated expectations. The second term is a correction for the fact that expectations are conditional on the seller being a low type; this term is negative and increases in magnitude as $\sigma_{j}$ increases because $\rho_{L}^{i}(b)-\rho_{\text {unc }}^{i}(b)$ and $V\left(\pi_{i}(g)\right)-V\left(\pi_{i}(b)\right)$ are increasing in $\sigma_{j}$. The last term is a correction for the fact that the bid following bad news is $v_{L}$ and not the pooling price $V\left(\pi_{i}(b)\right)$; this term is also negative and increases in magnitude as $\sigma_{j}$ increases because $\pi_{i}(b)$ is independent of $\sigma_{j}$ while $\rho_{L}^{i}(b)$ increases in $\sigma_{j}$. This establishes $(i)$.

For $(i i)$, when posteriors satisfy $\pi_{i}(b) \leq \bar{\pi} \leq \pi_{i}(g)$, we have

$$
Q_{H}^{i}\left(\sigma_{i}, \sigma_{j}, \cdot\right)=(1-\delta) c_{H}+\delta\left(\rho_{H}^{i}(b) c_{H}+\left(1-\rho_{H}^{i}(b)\right) V\left(\pi_{i}(g)\right)\right) .
$$

We can re-write this expression as

$$
\begin{aligned}
\delta^{-1}\left(Q_{H}^{i}\left(\sigma_{i}, \sigma_{j}, \cdot\right)-(1-\delta) c_{H}\right)=\underbrace{\rho_{\text {unc }}^{i}(b) V\left(\pi_{i}(b)\right)+\left(1-\rho_{\text {unc }}^{i}(b)\right) V\left(\pi_{i}(g)\right)}_{\text {unconditional expected value }} \\
+\underbrace{\left(\rho_{H}^{i}(b)-\rho_{\text {unc }}^{i}(b)\right)}_{-} \underbrace{\left(V\left(\pi_{i}(b)\right)-V\left(\pi_{i}(g)\right)\right)}_{-}+\rho_{H}^{i}(b) \underbrace{\left(c_{H}-V\left(\pi_{i}(b)\right)\right)}_{+} .
\end{aligned}
$$


The first term on the RHS is the unconditional expected value of the asset in $t=2$, which is independent of $\sigma_{j}$ by the law of iterated expectations. The second term is a correction for the fact that expectations are conditional on the seller being a high type; this term is positive and increases with $\sigma_{j}$ because $\rho_{\text {unc }}^{i}(b)-\rho_{H}^{i}(b)$ and $V\left(\pi_{i}(g)\right)-V\left(\pi_{i}(b)\right)$ are increasing in $\sigma_{j}$. The last term is a correction for the fact that the high type's payoff following bad news is $c_{H}$ and not the pooling price $V\left(\pi_{i}(b)\right)$; this term is also positive and increases with $\sigma_{j}$ because $\pi_{i}(b)$ is independent of $\sigma_{j}$ while $\rho_{H}^{i}(b)$ increases in $\sigma_{j}$. This establishes $(i i)$.

\section{PROOF OF LEMMA 5:}

Before proceeding with the proof, we introduce several definitions and make three observations. First, define $\sigma_{1}$ by

$$
\pi_{i}\left(g ; 0, \sigma_{1}\right)=\bar{\pi}
$$

and observe that $\sigma_{1} \in(0,1)$ if and only if $\lambda>\lambda_{1} \equiv 1-\pi \cdot \frac{1-\bar{\pi}}{1-\pi} \in(1-\pi, 1)$. Second, consider $\delta_{1, \lambda}<1$ defined by

$$
v_{L}=\left(1-\delta_{1, \lambda}\right) c_{L}+\delta_{1, \lambda}\left[\lambda \sigma_{1} v_{L}+\left(1-\lambda \sigma_{1}\right) c_{H}\right]
$$

where the right-hand side is equal to $Q_{L}^{i}\left(0, \sigma_{1}, 1\right)$ when $\delta=\delta_{1, \lambda}$. Note that $Q_{L}^{i}\left(0, \sigma_{1}, 1\right)>v_{L}$ if $\delta>\delta_{1} \equiv \sup _{\lambda \in(1-\pi, 1)} \delta_{1, \lambda} \in(0,1)$. Third, there exists $\lambda_{2} \in(1-\pi, 1)$ such that $Q_{L}^{i}(0,1,1)<v_{L}$ for $\lambda>\lambda_{2}$. This follows by continuity of $Q_{L}^{i}(0,1,1)$ in $\lambda$ and the fact that $\lim _{\lambda \rightarrow 1} Q_{L}^{i}(0,1,1)=(1-\delta) c_{L}+$ $\delta v_{L}<v_{L}$. In what follows, we assume that $\lambda$ and $\delta$ are sufficiently large meaning that $\lambda>\max \left\{\lambda_{1}, \lambda_{2}\right\}$ and $\delta>\delta_{1}$.

For $(i)$, it suffices to show that if $\sigma_{j}<\sigma_{1}$ the partial equilibrium for asset $i$ must feature $\pi_{i}(g)=\bar{\pi}$. Suppose not. Then it must be that $\pi_{i}(g)>\bar{\pi}$, which means that the bid in the second period is above $c_{H}$ following good news. Hence,

$$
Q_{L}^{i}>(1-\delta) c_{L}+\delta\left(\lambda \sigma_{j} v_{L}+\left(1-\lambda \sigma_{j}\right) c_{H}\right)>Q_{L}^{i}\left(0, \sigma_{1}, 1\right)>v_{L} .
$$

But if $Q_{L}^{i}>v_{L}$ then the optimal strategy for the low type is $\sigma_{i}=0$, which violates $\pi_{i}(g)>\bar{\pi}$.

For $(i i)$, we claim there exists $\sigma_{2} \in\left(\sigma_{1}, 1\right)$ such that $Q_{L}^{i}\left(0, \sigma_{2}, 1\right)=v_{L}$ and $Q_{L}^{i}\left(0, \sigma_{j}, 1\right) \geq v_{L}$ for $\sigma_{j} \in\left[\sigma_{1}, \sigma_{2}\right]$. Existence follows by continuity of $Q_{L}^{i}\left(0, \sigma_{j}, 1\right)$ in $\sigma_{j}$ for $\sigma_{j} \geq \sigma_{1}$ and our third observation (i.e., that $\left.Q_{L}^{i}(0,1,1)<v_{L}\right)$, while uniqueness follows from the fact that $Q_{L}^{i}\left(0, \sigma_{j}, 1\right)$ is decreasing in $\sigma_{j}$ for $\sigma_{j} \geq \sigma_{1}$ (see Lemma A.1 $(i)$ ). Note that $S\left(\sigma_{j}\right)$ must be zero on $\left[\sigma_{1}, \sigma_{2}\right]$.

For (iii), note that from our previous argument $Q_{L}^{i}\left(0, \sigma_{2}, 1\right)=v_{L}$ and $\pi_{i}\left(g ; 0, \sigma_{2}\right)>\bar{\pi}>\pi_{i}\left(b ; 0, \sigma_{2}\right)$ since $\sigma_{2}>\sigma_{1}$. Since $\pi_{i}\left(b ; \sigma_{i}, \sigma_{j}\right)$ is continuous in $\sigma_{i}$ and independent of $\sigma_{j}$ and because $Q_{L}^{i}\left(\sigma_{i}, \sigma_{j}, 1\right)$ is decreasing in $\sigma_{j}$ when $\pi_{i}(b)<\bar{\pi} \leq \pi_{i}(g)$ (see Lemma A.1 $(i)$ ), there exists $\sigma_{3} \in\left(\sigma_{2}, 1\right]$ such that the $S\left(\sigma_{j}\right)$ required to maintain $Q_{L}^{i}\left(S\left(\sigma_{j}\right), \sigma_{j}, 1\right)=v_{L}$ is strictly increasing on $\sigma_{j} \in\left[\sigma_{2}, \sigma_{3}\right]$ and satisfies $\pi_{i}\left(b ; S\left(\sigma_{j}\right), \sigma_{j}\right) \leq \bar{\pi}$. If $\sigma_{3}=1$, then $S$ is strictly increasing on $\left[\sigma_{2}, 1\right]$. But if $\sigma_{3}<1$ then $\pi_{i}\left(b ; S\left(\sigma_{3}\right), \sigma_{3}\right)=\bar{\pi}$ and $Q_{L}^{i}\left(\sigma_{3}, \sigma_{3}, 0\right)=v_{L}$, and for $\sigma_{j}>\sigma_{3}$ we have $Q_{L}^{i}\left(\sigma_{3}, \sigma_{j}, 0\right)<v_{L}$ by an argument analogous to that 
in proof of Lemma A.1 $(i)$. Since $Q_{L}^{i}$ is increasing in $\sigma_{i}, S\left(\sigma_{j}\right)$ cannot be lower than $S\left(\sigma_{3}\right)$. Hence, $S$ is nondecreasing on $\left[\sigma_{3}, 1\right]$.

\section{PROOF OF PROPOSITION 3:}

The proof that all equilibria involve strictly positive probability of trade at $t=1$ is in the text. We show here that all equilibria must be symmetric. In search of a contradiction, assume there exists an equilibrium in which $\sigma_{A}>\sigma_{B} \geq 0$. Then notice the following:

(i) The probability of bad news for seller $B$ is higher than that of bad news for seller $A$.

(ii) Conditional on bad news, beliefs must satisfy $\pi_{A}(b)>\pi_{B}(b)$ since

$$
\pi_{i}(b)=\frac{1}{1+\frac{\operatorname{Pr}\left(\theta_{j}=L \mid \theta_{i}=L\right)}{\operatorname{Pr}\left(\theta_{j}=L \mid \theta_{i}=H\right)} \cdot \frac{1-\pi_{\sigma_{i}}}{\pi_{\sigma_{i}}}}
$$

is increasing in $\sigma_{i}$ and is independent of $\sigma_{j}$.

(iii) Conditional on good news, beliefs must satisfy $\pi_{A}(g)>\pi_{B}(g)$ since

$$
\pi_{i}(g)=\frac{1}{1+\frac{1-\sigma_{j} \cdot \operatorname{Pr}\left(\theta_{j}=L \mid \theta_{i}=L\right)}{1-\sigma_{j} \cdot \operatorname{Pr}\left(\theta_{j}=L \mid \theta_{i}=H\right)} \cdot \frac{1-\pi_{\sigma_{i}}}{\pi_{\sigma_{i}}}}
$$

is more sensitive to own trading probability $\sigma_{i}$ than to $\sigma_{j}$ due to imperfect correlation.

Note that (i)-(iii) imply that $Q_{L}^{A}>Q_{L}^{B}$. Moreover, if $\sigma_{A} \in(0,1)$, it must be that $Q_{L}^{A}=v_{L}$ (by Lemma 4), which then implies that $Q_{L}^{B}<v_{L}$. This contradicts a necessary condition for a partial equilibrium for asset $B$ (also by Lemma 4 ). The symmetry in $\phi$ follows from monotonicity of $Q_{L}^{i}$ in $\phi_{i}$ whenever buyer mixing is part of an equilibrium.

\section{PROOF OF THEOREM 1:}

To prove existence of an equilibrium, it suffices to show there exists a $(\sigma, \phi) \in[0,1]^{2}$ such that equation (9) holds (i.e., $\left.Q_{L}(\sigma, \sigma, \phi)=v_{L}\right)$. Note that by varying $\sigma$ from 0 to $1, Q_{L}$ has a range $\left[(1-\delta) c_{L}+\delta v_{L},(1-\delta) c_{L}+\delta v_{H}\right]$. By continuity of $Q_{L}$ and Assumption 2, the intermediate value theorem gives the result.

(i) Low Trade (i.e., $\left.\pi_{i}(g)=\bar{\pi}>\pi_{i}(b)\right)$. - That there is at most one low trade equilibrium follows from the fact that the trading intensity $\sigma$ is fully pinned down by the requirement that $\pi_{i}(g)=\bar{\pi}$. Let $x$ be such that $\pi_{i}(g ; x, x)=\bar{\pi}$, and note that $x$ is decreasing in $\lambda$. As $\phi$ varies from 0 and $1, Q_{L}(x, x, \phi)$ varies continuously from $(1-\delta) c_{L}+\delta v_{L}$ to $(1-\delta) c_{L}+\delta\left(\lambda x \cdot v_{L}+(1-\lambda x) \cdot c_{H}\right)$. Hence, there exists a $\hat{\delta}_{\lambda}<1$, such that $Q_{L}(x, x, 1)=v_{L}$ when $\delta=\hat{\delta}_{\lambda}$. Clearly, a low trade equilibrium exists if $\delta>\hat{\delta}_{\lambda}$. Moreover, $\sup _{\lambda \in(1-\pi, 1)} \lambda x<1$. Hence $\bar{\delta} \equiv \sup _{\lambda \in(1-\pi, 1)} \hat{\delta}_{\lambda}<1$. 
(ii) High Trade (i.e., $\pi_{i}(g)>\bar{\pi}=\pi_{i}(b)$ ).- That there is at most one high trade equilibrium follows from the fact that the trading intensity $\sigma$ is fully pinned down by the requirement that $\pi_{i}(b)=\bar{\pi}$. Let $y$ be such that $\pi_{i}(b ; y, y)=\bar{\pi}$, and note that $y$ is increasing in $\lambda$. As $\phi$ varies from 0 to 1 , $Q_{L}(y, y, \phi)$ varies continuously from $(1-\delta) c_{L}+\delta\left(\lambda y \cdot v_{L}+(1-\lambda y)\right.$ - $\left.V\left(\pi_{i}(g)\right)\right)$ to $(1-\delta) c_{L}+\delta\left(\lambda y \cdot c_{H}+(1-\lambda y) \cdot V\left(\pi_{i}(g)\right)\right)$. Hence, we have $\lim _{\lambda \rightarrow 1} \lambda y=1$, it follows that the range of $Q_{L}$ converges to the interval $\left((1-\delta) c_{L}+\delta v_{L},(1-\delta) c_{L}+\delta c_{H}\right]$ as $\lambda$ goes to 1 . By Assumption 2, $v_{L}$ is inside this interval. This establishes the existence of a threshold $\bar{\lambda}_{\delta}$ such that the high trade equilibrium exists whenever $\lambda>\bar{\lambda}_{\delta}$.

Notice that we can already conclude that the low and the high trade equilibria coexist whenever $\delta>\bar{\delta}$ and $\lambda>\bar{\lambda}_{\delta}$. We are thus left to show that there are at most two medium trade equilibria, and that there is only one medium trade equilibrium when these parametric conditions hold.

(iii) Medium Trade (i.e., $\left.\pi_{i}(g)>\bar{\pi}>\pi_{i}(b)\right)$. - We first show that there can be at most two medium trade equilibria. For the remainder of the proof, let $r$ be a candidate trading probability in a medium trade equilibrium (i.e., such that $\pi(b ; r, r)<\bar{\pi}<\pi(g ; r, r))$. Then,

$$
Q_{L}(r, r, \cdot)=(1-\delta) c_{L}+\delta\left[f(r) v_{H}+(1-f(r)) v_{L}\right]
$$

where

$$
f(r)=\left(\frac{1}{1-\rho_{L}(b)}+\frac{1-r}{1-\rho_{H}(b)} \cdot \frac{1-\pi}{\pi}\right)^{-1},
$$

and $\rho_{\theta}(b)=r \operatorname{Pr}\left(\theta_{j}=L \mid \theta_{i}=\theta\right)$ for $\theta \in\{L, H\}$. It can be shown that $f(r)$ is continuously differentiable and the equation $f^{\prime}(r)=0$ has at most one solution in $(0,1)$, which implies that $Q_{L}$ is equal to $v_{L}$ for at most two values of $r$. This establishes that there can be at most two medium trade equilibria.

Suppose that the low and the high trade equilibria coexist. For existence, let $x, y$ be defined by

$$
\pi_{i}(b ; y, y)=\bar{\pi}=\pi_{i}(g ; x, x) .
$$

From the monotonicity of posteriors in the trading probability, we have $r \in(x, y)$. Since the low and the high trade equilibria coexist, there exist $\phi^{\prime}, \phi^{\prime \prime} \in(0,1)$ such that $Q_{L}\left(x, x, \phi^{\prime}\right)=Q_{L}\left(y, y, \phi^{\prime \prime}\right)=v_{L} \cdot{ }^{24}$ Now, note that $\lim _{r \downarrow x} Q_{L}(r, r, \cdot)=Q_{L}(x, x, 1)$ and $\lim _{r \uparrow y} Q_{L}(r, r, \cdot)=Q_{L}(y, y, 0)$. But then, because $Q_{L}(x, x, 1)>Q_{L}\left(x, x, \phi^{\prime}\right)=Q_{L}\left(y, y, \phi^{\prime \prime}\right)>Q_{L}(y, y, 0)$, the intermediate value theorem implies that there must exist an $r^{\prime}$ such that $Q_{L}\left(r^{\prime}, r^{\prime}, \cdot\right)=v_{L}$.

\footnotetext{
${ }^{24}$ We ignore non-generic cases where either $\phi^{\prime}=1$ or $\phi^{\prime \prime}=0$, which are ruled out when $\delta>\bar{\delta}$ and $\lambda>\bar{\lambda}_{\delta}$.
} 
For uniqueness, we proceed by contradiction. Suppose that there also exists an $r^{\prime \prime}$ such that $r^{\prime \prime}>r^{\prime}$ and $Q_{L}\left(r^{\prime \prime}, r^{\prime \prime}, \cdot\right)=v_{L}$. Recall that $Q_{L}$ intersects $v_{L}$ for at most two values of $r$, namely $r^{\prime}$ and $r^{\prime \prime}$, whenever $\pi(b ; r, r)<\bar{\pi}<\pi(g ; r, r)$. Then either (i) $Q_{L}(r, r, \cdot)<v_{L}$ for all $r \in\left(x, r^{\prime}\right) \cup\left(r^{\prime \prime}, y\right)$ or $\left(\right.$ ii) $Q_{L}(r, r, \cdot)>v_{L}$ for all $r \in\left(x, r^{\prime}\right) \cup\left(r^{\prime \prime}, y\right)$. But recall that $\lim _{r \downarrow x} Q_{L}(r, r, \cdot)=Q_{L}(x, x, 1)>Q_{L}\left(x, x, \phi^{\prime}\right)=v_{L}$ which contradicts (i), and $\lim _{r \uparrow y} Q_{L}(r, r, \cdot)=Q_{L}(y, y, 0)<Q_{L}\left(y, y, \phi^{\prime \prime}\right)=v_{L}$ which contradicts (ii). Thus, there exists one medium trade equilibrium.

The convergence of the trading probability to $\bar{\sigma}$, as $\lambda$ goes to $1-\pi$, follows from the following observation. In any equilibrium, $\pi_{i}(b) \leq \bar{\pi}$ $\leq \pi_{i}(g)$ (see discussion preceding Theorem 1). Hence, by continuity of posteriors in $\lambda$ and $\sigma$, we have $\lim _{\lambda \rightarrow 1-\pi} \pi_{i}(b)=\lim _{\lambda \rightarrow 1-\pi} \pi_{i}(g)$ $=\lim _{\lambda \rightarrow 1-\pi} \pi_{\sigma}=\bar{\pi}$, and therefore $\lim _{\lambda \rightarrow 1-\pi} \sigma=\bar{\sigma}$. Let us now consider the uniqueness argument.

Consider $\delta_{0} \in(0,1)$ defined by

$$
v_{L}=\left(1-\delta_{0}\right) c_{L}+\delta_{0}\left((1-\pi) \bar{\sigma} \cdot v_{L}+(1-(1-\pi) \bar{\sigma}) \cdot c_{H}\right) .
$$

We now show that a sufficient condition for the equilibrium to be unique for $\lambda$ close to $1-\pi$ is that $\delta \neq \delta_{0}$ (hence our qualifier that the equilibrium is generically unique). As $\lambda$ approaches $1-\pi$, in equilibrium posteriors converge as well. In particular, we have that for $r$ satisfying $\pi(b ; r, r)$ $<\bar{\pi}<\pi(g ; r, r)$ :

$$
\begin{aligned}
& \lim _{\lambda \rightarrow 1-\pi} Q_{L}(r, r, \phi) \\
& \quad=(1-\delta) c_{L}+\delta\left[(1-\pi) \bar{\sigma} \cdot v_{L}+(1-(1-\pi) \bar{\sigma}) \cdot c_{H}\right] .
\end{aligned}
$$

But if $\delta \neq \delta_{0}$, then for $\lambda$ close to $1-\pi$, a medium trade equilibrium cannot exist, because otherwise we cannot have $Q_{L}=v_{L}$ as required by (9). Thus, we have ruled out the medium trade equilibrium. But from our earlier arguments, the low and the high trade equilibrium generically do not coexist when the medium trade equilibrium does not exist.

\section{PROOF OF PROPOSITION 4:}

See text for the arguments for the high and low trade equilibria. For the medium trade equilibria, the proof is by example (see Figure 4, panel A).

\section{PROOF OF PROPOSITION 5:}

The welfare of the high type in the low trade equilibrium is $c_{H}$. Also, note that in the medium and high trade equilibria, we have that the posterior following good news satisfies $\pi_{i}(g)>\bar{\pi}$, which implies that in those equilibria the high type's welfare is strictly above $c_{H}$. Thus, welfare in the high or medium trade equilibria is higher than in the low trade equilibrium. We are left to rank the welfare in the medium and high trade equilibria and demonstrate the comparative statics results. 
For the welfare ranking, note that $\pi_{i}\left(b ; \sigma^{\text {med }}, \sigma^{\text {med }}\right)<\bar{\pi}=\pi_{i}\left(b ; \sigma^{\text {high }}, \sigma^{\text {high }}\right)$, and $\sigma^{\text {high }}>\sigma^{\text {med }}$. We know that $Q_{H}^{i}$ is increasing in $\sigma_{i}$ and in $\sigma_{j}$ by Lemma A.1 (ii) as both increase from $\sigma^{\text {med }}$ to $\sigma^{\text {high }}$. Hence, $Q_{H}^{\text {high }}>Q_{H}^{\text {med }}$.

For the comparative statics, note that in the low trade equilibrium $\pi_{i}(g)=\bar{\pi}$; therefore, $Q_{H}^{\text {low }}=c_{H}$ for all $\lambda$. In high trade equilibrium, we have $\pi_{i}(b)=\bar{\pi}$, which implies that the equilibrium trading intensity $\sigma^{\text {high }}$ is increasing with $\lambda$. Fixing $\sigma$ such that $\pi_{i}(b)=\bar{\pi}, Q_{H}^{i}$ increases with $\lambda$, since $\rho_{H}^{i}(b)$ and $\pi_{i}(b)$ decrease with $\lambda, \pi_{i}(g)$ increases with $\lambda$, and because

$$
Q_{H}^{i}=(1-\delta) c_{H}+\delta\left(\rho_{H}^{i}(b) c_{H}+\left(1-\rho_{H}^{i}(b)\right) V\left(\pi_{i}(g)\right)\right.
$$

for all such $\lambda$. But using the argument above, we know that $Q_{H}^{i}$ increases with $\sigma$ as it increases to the new equilibrium value $\sigma^{\text {high }}$. Hence, $Q_{H}^{\text {high }}$ increases with $\lambda$. Finally, that welfare in the medium trade equilibrium can be decreasing with $\lambda$ is illustrated in Figure 4.

\section{PROOF OF PROPOSITION 6:}

In an opaque market, the equilibrium trading probability is $\bar{\sigma}$ satisfying $\pi_{\bar{\sigma}}=\bar{\pi}$ as in Proposition 1, and the high type's payoff is $c_{H}$. With post-trade transparency, all buyers observe all transactions and thus, the set of equilibria are the same as in Theorem 1 and welfare is as in Proposition 5. Thus, there are multiple equilibria (i.e., low, medium, high trade) if $\lambda>\bar{\lambda}_{\delta}$, and the equilibrium is unique (and given by the low trade equilibrium) when $\lambda$ is sufficiently small. This follows from the fact that the low trade equilibrium exists when $\delta>\bar{\delta}$ and that the equilibrium is unique for $\lambda$ sufficiently small.

When $\lambda>\bar{\lambda}_{\delta}$, trade volume can increase or decrease. This follows from the observation that $\sigma^{\text {low }}<\bar{\sigma}<\sigma^{\text {high }}$ since posterior beliefs are monotonic in $\sigma, \pi_{i}(b)<\pi_{\sigma}<\pi_{i}(g)$, and $\pi_{i}\left(b ; \sigma^{h i g h}, \sigma^{h i g h}\right)=\pi_{i}\left(g ; \sigma^{\text {low }}, \sigma^{\text {low }}\right)=\bar{\pi}$. In the medium and high trade equilibria, welfare is greater than in the low trade equilibrium (see Proposition 5), which is equal to welfare when the market is opaque. Thus, welfare increases if multiple equilibria exist and agents play the medium or high trade equilibrium, but remains unchanged if the low trade equilibrium is played, which completes the argument for $(i)$.

To complete the argument for $(i i)$, recall that when $\lambda$ is sufficiently small and $\delta>\bar{\delta}$, the unique equilibrium involves $\pi_{i}(g)=\bar{\pi}$ (hence $\sigma<\bar{\sigma}$ ) and $Q_{H}^{i}=c_{H}$. Hence, introducing post-trade transparency reduces trade volume while welfare remains unchanged.

\section{PROOF OF PROPOSITION 7:}

If investors are naïve, they are unaware there is an additional informed buyer in the market and thus they bid as if they were in an opaque market. Since there are many naïve investors and one insider, the second highest bid is always determined by the naïve investors. Thus the equilibrium distribution of bids and trading probability $\sigma$ are as in the case without information spillovers (as in Proposition 1), which means that the welfare of the seller and the total welfare also coincide, and are the same as in the low trade equilibrium in Proposition 5. 
For the insider, it is a weakly dominant strategy to bid his expected value when it is above $c_{H}$ and $v_{L}$ otherwise. When there is good news his expected value is strictly above $c_{H}$. When there is bad news his expected value is below $c_{H}$, and thus he will bid at most $v_{L}$.

Given the equilibrium bidding and trading strategies described above, the informed buyer always wins the auction when he observes good news and makes zero profit following bad news. Hence, the informed buyer makes rents since the fact he observed good news raises his posterior expected value above the price he pays (either $v_{L}$ or $c_{H}$ ). When the naïve investors win the auction, it must have been that the insider observed bad news. They fall prey to the winner's curse. The true expected value of the asset is below $c_{H}$ and thus they make losses in expectation. Since total surplus and the sellers' welfare is identical to the low trade equilibrium, it immediately follows that the rents made by the informed are exactly offset by the losses of the uninformed.

\section{PROOF OF PROPOSITION 8:}

As in the case with naïve investors, it is a weakly dominant strategy for the insider to offer his expected value of the asset (i.e., $\left.V\left(\pi_{i}(z)\right)\right)$ if it is above $c_{H}$ and $v_{L}$ otherwise. In an opaque market, sophisticated investors do not observe news, but they are aware that if they bid solely based on their interim posterior, they would only win when the informed buyer observes bad news (as detailed in the proof of Proposition 7). The only way to avoid these loses is by bidding as if they observed bad news. This way they never overbid for the asset. If $\pi_{i}(b)>\bar{\pi}$, then the bid in the second period would always be above $c_{H}$ and the low type would not be willing to trade in the first period. Hence, $\pi_{i}(b) \leq \bar{\pi}$ and sophisticated investors can only bid $c_{H}$ or something weakly less than $v_{L}$. Since there is only one insider, the price will again be set by the investors.

Given the bidding behavior described above, our condition for equilibrium becomes

$$
v_{L}=(1-\delta) c_{L}+\delta F_{L}\left(\pi_{i}(b), \phi_{i}\right)
$$

Assumptions 1 and 2 imply that the only possible solution must involve $\pi_{i}(b)=\bar{\pi}$. Hence, there is a unique equilibrium $\sigma$, which is such that $\pi_{i}(b ; \sigma, \sigma)=\bar{\pi}$. Notice that this equilibrium involves a higher $\sigma$ than in the low trade equilibrium since $\pi_{i}(b)<\pi_{\sigma_{i}}$.

Clearly, both the low type and the sophisticated investors have the same payoffs as in the low trade equilibrium of Theorem 1 (i.e., $v_{L}$ and 0 ). The high type's payoff is also the same as in the low-trade equilibrium since he gets $c_{H}$ at $t=2$ regardless of the realization of news. The insider, however, makes positive expected profits since after observing good news he buys the asset for $c_{H}$ but when the expected value is $V\left(\pi_{i}(g)\right)>c_{H}$. 


\section{REFERENCES}

Adelino, Manuel, Kristopher Gerardi, and Barney Hartman-Glaser. 2016. "Are Lemons Sold First? Dynamic Signaling in the Mortgage Market.” https://papers.ssrn.com/sol3/papers.cfm?abstract_ id $=2811560$ (accessed November 2016).

-Akerlof, George A. 1970. "The Market for 'Lemons': Quality Uncertainty and the Market Mechanism." Quarterly Journal of Economics 84 (3): 488-500.

Alti, Aydogan, Joseph Engelberg, and Christopher A. Parsons. 2011. "Information Spillovers and House Prices.” https://papers.ssrn.com/sol3/papers.cfm?abstract_id=1786824 (accessed November 2016).

Asquith, Paul, Thom Covert, and Parag Pathak. 2013. "The Effects of Mandatory Transparency in Financial Market Design: Evidence from the Corporate Bond Market." National Bureau of Economic Research Working Paper 19417.

Asriyan, Vladimir, William Fuchs, and Brett Green. 2016. "Information Aggregation in Dynamic Markets with Adverse Selection.” https://papers.ssrn.com/sol3/papers.cfm?abstract_id=1786824 (accessed November 2016).

- Bessembinder, Hendrik, and William Maxwell. 2008. "Market Transparency and the Corporate Bond Market." Journal of Economic Perspectives 22 (2): 217-34.

- Bessembinder, Hendrik, William Maxwell, and Kumar Venkataraman. 2006. "Market Transparency, Liquidity Externalities, and Institutional Trading Costs in Corporate Bonds." Journal of Financial Economics 82 (2): 251-88.

-Cespa, Giovanni, and Xavier Vives. 2015. "The Beauty Contest and Short-Term Trading." Journal of Finance 70 (5): 2099-2154.

-Daley, Brendan, and Brett Green. 2012. "Waiting for News in the Market for Lemons." Econometrica 80 (4): 1433-1504.

-Daley, Brendan, and Brett Green. 2016. "An Information-Based Theory of Time-Varying Liquidity." Journal of Finance 71(2): 809-70.

Drugov, Mikhail. 2010. "Contracts with Informational Externalities.” Unpublished.

Drugov, Mikhail. 2014. "Bargaining with Informational Externalities in a Market Equilibrium." https:// papers.ssrn.com/sol3/papers.cfm?abstract_id=2501493 (accessed November 2016).

Duffie, Darrell, Piotr Dworczak, and Haoxiang Zhu. Forthcoming. "Benchmarks in Search Markets." Journal of Finance.

DEdwards, Amy K., Lawrence E. Harris, and Michael S. Piwowar. 2007. "Corporate Bond Market Transaction Costs and Transparency." Journal of Finance 62 (3): 1421-51.

-Fuchs, William, Aniko Öry, and Andrzej Skrzypacz. 2016. "Transparency and Distressed Sales under Asymmetric Information." Theoretical Economics 11 (3): 1103-44.

Goldstein, Michael A., Edith S. Hotchkiss, and Erik R. Sirri. 2007. "Transparency and Liquidity: A Controlled Experiment on Corporate Bonds." Review of Financial Studies 20 (2): 235-73.

Gordon, Jacques N. 2012. "Real Estate Transparency." Samuel Zell and Robert Lurie Real Estate Center Working Paper 734.

Healy, Paul M., and Krishna G. Palepu. 2001. "Information Asymmetry, Corporate Disclosure, and the Capital Markets: A Review of the Empirical Disclosure Literature." Journal of Accounting and Economics 31 (1-3): 405-40.

- Hörner, Johannes, and Nicolas Vieille. 2009. "Public vs. Private Offers in the Market for Lemons." Econometrica 77 (1): 29-69.

Kaya, Ayça, and Kyungmin Kim. 2015. "Trading Dynamics with Private Buyer Signals in the Market for Lemons." https://55f4a62f-a-62cb3ala-s-sites.googlegroups.com/site/kyungminteddykim/ papers-2/Dynamics_Lemon_062015.pdf? (accessed November 2016).

-Kremer, Ilan, and Andrzej Skrzypacz. 2007. "Dynamic Signaling and Market Breakdown." Journal of Economic Theory 133 (1): 58-82.

Kurlat, Pablo, and Johannes Stroebel. 2015. "Testing for Information Asymmetries in Real Estate Markets." Review of Financial Studies 28 (8): 2429-61.

-Kyle, Albert S. 1985. "Continuous Auctions and Insider Trading." Econometrica 53 (6): 1315-35.

Learner, Heider. 2011. "An Examination of Transparency in European Bond Markets." CFA Institute Technical Report 2011 (5).

-Milgrom, Paul, and Nancy Stokey. 1982. "Information, Trade and Common Knowledge." Journal of Economic Theory 26 (1): 17-27.

Naik, Narayan Y., Anthony Neuberger, and S. Viswanathan. 1999. "Trade Disclosure Regulation in Markets with Negotiated Trades." Review of Financial Studies 12 (4): 873-900.

National Association of Securities Dealers (NASD). 2005. NASD's Fully Implemented 'TRACE' Brings Unprecedented Transparency to Corporate Bond Market. Washington, DC: Financial Industry Regulatory Authority. 
Nöldeke, Georg, and Eric van Damme. 1990. "Signalling in a Dynamic Labour Market." Review of Economic Studies 57 (1): 1-23.

Richardson, Heather, and Leonard V. Zumpano. 2012. "Further Assessment of the Efficiency Effects of Internet Use in Home Search.” Journal of Real Estate Research 34 (4): 517-48.

Swinkels, Jeroen M. 1999. "Education Signalling with Preemptive Offers." Review of Economic Studies 66 (4): 949-70.

- Verrecchia, Robert E. 2001. "Essays on Disclosure.” Journal of Accounting and Economics 32 (1-3): 97-180. 